\title{
Pseudoscalar decays to gauge bosons at the LHC and at a future $100 \mathrm{TeV}$ collider
}

\author{
Abdesslam Arhrib, ${ }^{1, *}$ Rachid Benbrik, ${ }^{2, \dagger}$ Jaouad El Falaki, ${ }^{1,3, \ddagger}$ Marco Sampaio, ${ }^{4,5, \S}$ and Rui Santos ${ }^{6,7, \|}$ \\ ${ }^{1}$ Département de Mathématiques, Faculté des Sciences et Techniques, \\ Université Abdelmalek Essaadi, B. 416 Tangier, Morocco \\ ${ }^{2}$ LPHEA, MSISM Team Départment of Physics, Faculté Polydisciplinaire de Safi, \\ Sidi Bouzid, BP 4162 Safi, Morocco \\ ${ }^{3}$ Center for Fundamental Physics, Zewail City of Science and Technology, \\ Sheikh Zayed,12588 Giza, Egypt \\ ${ }^{4}$ Departamento de Física da Universidade de Aveiro, Campus de Santiago, 3810-183 Aveiro, Portugal \\ ${ }^{5}$ CIDMA-Center for Research \& Development in Mathematics and Applications, \\ Campus de Santiago, 3810-183 Aveiro, Portugal \\ ${ }^{6}$ ISEL_Instituto Superior de Engenharia de Lisboa, Instituto Politécnico de Lisboa, \\ 1959-007 Lisboa, Portugal \\ ${ }^{7}$ Centro de Física Teórica e Computacional, Faculdade de Ciências, Universidade de Lisboa, \\ Campo Grande, Edifício, C8 1749-016 Lisboa, Portugal
}

(Received 20 September 2018; published 27 February 2019)

\begin{abstract}
We discuss the search for a $C P$-odd scalar decaying into gauge bosons in the frameworks of a $C P$-conserving two-Higgs doublet model (2HDM) and of a $2 \mathrm{HDM}$ extended with a vectorlike quark (VLQ) at the Large Hadron Collider and at a future $100 \mathrm{TeV}$ collider. The rate of decay of a pseudoscalar to $Z$ bosons could be important to ascertain the $C P$ nature of the scalars in the model. In the $2 \mathrm{HDM} A \rightarrow Z Z$ will be extremely hard to detect even at a future $100 \mathrm{TeV} p p$ collider while in the 2HDM+VLQ this decay can be probed even during the present LHC run. We further discuss all decays of the pseudoscalar into gauge bosons at the LHC and at a future $100 \mathrm{TeV}$ collider in the alignment limit where the lightest scalar is the $125 \mathrm{GeV}$ Higgs with SM-like couplings to the fermions and gauge bosons.
\end{abstract}

DOI: 10.1103/PhysRevD.99.035043

\section{INTRODUCTION}

After the discovery of the Higgs boson by the ATLAS [1] and CMS [2] Collaborations at the Large Hadron Collider (LHC), the high-energy physics community focused on the search for signs of extended scalar sectors [3]. Such extended sectors, with extra Higgs singlets, doublets, or triplets, are a common feature of several beyond the standard model (BSM) models. Finding a new scalar would be a clear signal of BSM physics with extended Higgs sectors. Two-Higgs doublet models (2HDM) [4], both in their $C P$-conserving and $C P$-violating versions, have been

\footnotetext{
*aarhrib@gmail.com

r.benbrik@uca.ac.ma

¥jaouad.elfalaki@gmail.com

\$msampaio@ua.pt

"rasantos@fc.ul.pt
}

Published by the American Physical Society under the terms of the Creative Commons Attribution 4.0 International license. Further distribution of this work must maintain attribution to the author(s) and the published article's title, journal citation, and DOI. Funded by SCOAP. used as benchmark models to search for new scalars at the LHC. The 2HDM has four extra degrees of freedom with two extra neutral scalars and two charged scalars. In the $C P$-conserving version of the model, the two neutral states are $h$ and $H$ ( $C P$-even) and $A$ ( $C P$-odd $)$ while in the $C P$-violating version the three neutral states are a mixture of $C P$-even and $C P$-odd states and are referred to as $h_{1}, h_{2}$ and $h_{3}$. In this work, we focus on the production of the pseudoscalar via gluon fusion plus $b \bar{b}$ initiated process with the subsequent decay to gauge bosons, with focus on $A \rightarrow \gamma \gamma$ and $A \rightarrow Z Z$. Although $A \rightarrow \gamma \gamma$ is a loop-induced process, it is nevertheless competitive with other final states like $\tau^{+} \tau^{-}$in large portions of the parameter space of the model. Hence, a pseudoscalar could first be detected in the two photon final state. However, even if $A$ is discovered in some other final states, the remaining possible decays have either to be confirmed or excluded. This study will therefore give us further information on the model.

Previous works have discussed the pseudoscalar decays into gauge bosons in a variety of models such as the 2HDM [5-7], the 2HDM with a sequential fourth generation of quarks [8] and in Supersymetric Models [9]. In this work, our main focus either diverges or completes the previous 
studies. In terms of completing and/or updating the studies, we discuss how both $A \rightarrow \gamma \gamma$ and $A \rightarrow Z Z$ are affected by the latest experimental searches at the LHC together with the most relevant and up-to-date experimental and theoretical constraints. We then move to discuss what is expected by the end of the LHC and also at a future $100 \mathrm{TeV} p p$ collider. We include for the first time the study of the processes in an extension of the 2HDM with the addition of vectorlike quarks (VLQ) [10-12]. The extra quark loops present both in the production and in the decay may lead to a significant enhancement of the rates relative to the 2HDM. Therefore, the rate $p p \rightarrow A \rightarrow Z Z$ can differ by several orders of magnitude in the $2 \mathrm{HDM}$ and in the $2 \mathrm{HDM}+\mathrm{VLQ}$ which shows that loop processes can vary by orders of magnitude in two simple extensions of the SM. This extension of the $2 \mathrm{HDM}$ is used to show that the range of possible variation of the number of events produced at loop level can be very similar to the ones produced at tree-level in a model with the same potential but with extra fermion content.

Most importantly, the following question was not asked in previous works: to what extent can we say if we are indeed seeing a pseudoscalar? We want to understand what to expect if a scalar is found decaying into a $Z Z$ final state. Therefore, not only we discuss the event rates of a $p p \rightarrow$ $A \rightarrow Z Z$ in two different models, but also discuss the different possibilities of distinguishing a scalar with definite $C P$ from a $C P$-violation one in the context of $2 \mathrm{HDMs}$.

ATLAS and CMS have shown that if the $125 \mathrm{GeV}$ Higgs has a definite $C P$, then it does not have $C P=-1$. The search for $C P$ violation in the $H Z Z$ vertex was and is still being performed at the LHC $[13,14]$ using the method described in $[15,16]$. However, it is very important to stress that in models such as $C P$-violating versions of the $2 \mathrm{HDM}$, as in all $C P$-violating extensions with singlets and doublets, the $H Z Z$ has its origin in the Lagrangian term $\left(D_{\mu} \phi\right)^{\dagger} D^{\mu} \phi$, where $\phi$ is an $S U(2)$ doublet, and therefore it has the simple SM Lorentz structure proportional to $g_{\mu \nu}$ at tree-level. Therefore, the measurements of the effective operators in $[13,14]$ can at most be used to constrain these models at loop level.

One way to look for signs of $C P$ violation at the $\mathrm{LHC}$ is in the decays to $Z Z$ [17]. Several classes of processes may hint a signal of $C P$ violation. One such example is the combined observation of the three decays $h_{2} \rightarrow h_{1} Z, h_{2} \rightarrow$ $Z Z$ and $h_{1} \rightarrow Z Z$, where $h_{1}$ is the $125 \mathrm{GeV}$ Higgs. Except for the already measured $h_{1} \rightarrow Z Z$, the other two processes that occur at tree-level in a $C P$-violating model, can be mistaken by the loop processes $A \rightarrow h Z$ and $A \rightarrow Z Z$ from the corresponding $C P$-conserving model. In the alignment limit, where all $h_{1} / h$ couplings to the SM particles mimic the SM ones, $A \rightarrow h Z$ is zero at tree-level. Also, $A \rightarrow Z Z$ is a loop-induced process and therefore very small. Hence, if a new scalar is found, its $C P$-numbers could be hard if not at all impossible to determine with this method if the rates are too small.
Another way to look for a similar effect in a single process is to look for anomalous ( $\mathrm{CP}$-violating) coupling in the triple gauge bosons vertices [18-21]. The structure of the off-shell $Z^{*} Z Z$ vertex, has terms that are only nonzero if $C P$ violation is present in the model. This was calculated for the particular case of a complex version of the 2HDM (the C2HDM) [22,23] (see [24] for a recent review on the $\mathrm{C} 2 \mathrm{HDM}$ ) and also for an extension of the 2HDM with an extra singlet where $C P$ violation only exists in the dark scalar sector [25] and therefore looking for the three decays that compose the one-loop contribution to $Z^{*} Z Z$ is not an option. In this model, the only loop diagram that contributes to $Z^{*} Z Z$ involves all vertices $h_{i} Z Z, i=1,2,3$. The present LHC results probe the $C P$-violating term $f_{4}^{Z}$ to order $\approx 10^{-3}$ [26-28], whereas the typical magnitude of the $f_{4}^{Z}$ term (both real and imaginary parts) is $\approx 10^{-5}$. Recently, a study for the LHC [29] has shown that for the LHC@14 TeV an increase in luminosity from $36 \mathrm{fb}^{-1}$ to $1000 \mathrm{fb}^{-1}$ implies an improvement on the measurement by only a factor of 2, even with the inclusion of asymmetries which were shown not to have a significant impact in the limit. Therefore, the typical magnitudes will probably be out of reach even for the high luminosity stage, although no study is still available. There are also no studies for a future $100 \mathrm{TeV} p p$ collider.

The previous discussion shows that probing the $C P$ nature of a new scalar is going to be extremely hard. In fact, if a new scalar is found in the $Z Z$ channel, especially with low rates, we believe the only possibility to probe its $C P$ nature is through the Yukawa couplings. In this case, the Yukawa couplings have to be large enough to allow this distinction, which is certainly possible in models like the $2 \mathrm{HDM}$ or the C2HDM, where even if $g_{H Z Z}$ is small, Yukawa couplings can still be large. The discussion of $C P$ violation in the Yukawa couplings will appear in Sec. VII and in the conclusions.

The paper is organized as follows. In the next section, we discuss the 2HDM setup and list the theoretical and experimental constraints we will be using. In Sec. III, we present the 2HDM extended by an up-type vectorlike quark (2HDM+T). In Sec. IV, we present our results for the $2 \mathrm{HDM}$, while in Sec. V results for the $2 \mathrm{HDM}+\mathrm{T}$ are presented for the LHC Run 2. A discussion on the prospects for a future $100 \mathrm{TeV}$ collider are examined in Sec. VI. Finally, in Sec. VII, we address the problem of the contribution of these searches to understand the $C P$ nature of a new scalar. Our conclusions are presented in the last section.

\section{THE TWO-HIGGS DOUBLET MODEL}

The 2HDM was proposed by T.D. Lee [4] in an attempt to explain the matter anti-matter asymmetry of the Universe through the addition of an extra source of $C P$ violation. In this work, we discuss the $C P$-conserving version of the 
model that contains two $C P$-even states denoted by $h$ (the lightest) and $H$, one $C P$-odd state denoted by $A$ and two charged states, $H^{ \pm}$. As tree-level flavor changing neutral currents (FCNC) are very constrained by experiments, we impose a $\mathbb{Z}_{2}$ symmetry $\Phi_{1} \rightarrow \Phi_{1}$ and $\Phi_{2} \rightarrow-\Phi_{2}$ on the scalar fields. The resulting Higgs potential (softly broken by the dimension two term $m_{12}^{2}$ ) can be written as

$$
\begin{aligned}
\mathcal{V}= & m_{11}^{2} \Phi_{1}^{\dagger} \Phi_{1}+m_{22}^{2} \Phi_{2}^{\dagger} \Phi_{2}-\left(m_{12}^{2} \Phi_{1}^{\dagger} \Phi_{2}+\text { H.c. }\right) \\
& +\frac{1}{2} \lambda_{1}\left(\Phi_{1}^{\dagger} \Phi_{1}\right)^{2}+\frac{1}{2} \lambda_{2}\left(\Phi_{2}^{\dagger} \Phi_{2}\right)^{2}+\lambda_{3} \Phi_{1}^{\dagger} \Phi_{1} \Phi_{2}^{\dagger} \Phi_{2} \\
& +\lambda_{4} \Phi_{1}^{\dagger} \Phi_{2} \Phi_{2}^{\dagger} \Phi_{1}+\left[\frac{1}{2} \lambda_{5}\left(\Phi_{1}^{\dagger} \Phi_{2}\right)^{2}+\text { H.c. }\right] .
\end{aligned}
$$

Choosing real vacuum expectation values (VEVs), $v_{1}$ and $v_{2}$ and demanding $m_{12}^{2}$ and $\lambda_{5}$ to be real as well, the potential is $C P$-conserving. One should note that the $C P$ conserving minimum of any $2 \mathrm{HDM}$ is stable at tree-level, that is, any other stationary point, if it exists, is a saddle point $[30,31]$. Still two $C P$-conserving minima can coexist but the existence of a global minimum can be easily enforced by a simple condition [32,33]. The free independent parameters are the four masses, $m_{h}, m_{H}, m_{A}$ and $m_{H^{ \pm}}$, the soft breaking parameter $m_{12}^{2}$, the angle $\tan \beta=v_{2} / v_{1}$ and the rotation angle $\alpha$ that diagonalizes the $C P$-even mass matrix.

When we impose that no tree-level FCNCs are present in the theory by extending the $\mathbb{Z}_{2}$ symmetry [34,35] to the Yukawa sector, we end up with four independent versions of the model. These are type I-only $\Phi_{2}$ couples to all fermions; type II $-\Phi_{2}$ couples to up-type quarks and $\Phi_{1}$ couples to charged leptons and down-type quarks; flipped or type $\mathrm{Y}-\Phi_{2}$ couples to charged leptons and up-type quarks and $\Phi_{1}$ couples to down-type quarks; lepton specific or type $\mathrm{X}-\Phi_{2}$ couples to quarks and $\Phi_{1}$ couples to charged leptons.

The scan in the 2HDM parameter space was performed fixing $m_{h}=125 \mathrm{GeV}, \sin (\beta-\alpha)=1$, since a small misalignment has no phenomenological consequences on the $C P$-odd decay, and $m_{H^{ \pm}}=m_{H}=600 \mathrm{GeV}$ and varying $m_{A}, \tan \beta$ and $m_{12}^{2}$ in the allowed parameter space. This is the exact alignment limit and it is in agreement with the most relevant experimental and theoretical constraints:

(i) The potential is bounded from below at treelevel [36,37];

(ii) Perturbative unitarity is enforced [38-40] to the quartic couplings of the Higgs potential;

(iii) The parameter space complies with electroweak precision observables [41] via $S$ and $\mathrm{T}$ parameters [42-46] because $m_{H^{ \pm}}=m_{H}$ and $\cos (\beta-\alpha)=0$ [45];

(iv) Collider bounds from LEP, Tevatron and from LHC Run 1 are taken into account. Since we work in the alignment limit, $\sin (\beta-\alpha)=1$, automatic agreement with the constraints on the Higgs couplings to the other SM particles is attained, because all Higgs couplings become SM-like. Regarding the searches, the tree-level decays to gauge bosons of both $H$ and $A$ are forbidden. The decays to fermions are considered and of particular relevance are the bounds arising from the search $p p \rightarrow A \rightarrow \tau^{+} \tau^{-}$[47-49]. These imply that in the type II $2 \mathrm{HDM}$, the values of $\tan \beta$ cannot be too large especially for low $m_{A}$ bounds. Therefore we choose to take $\tan \beta<10$ in the entire mass range for type II. However, one should note that the largest cross sections are the gluon fusion ones, with the maximum value for $\tan \beta=1$. Also the $\operatorname{BR}(A \rightarrow \gamma \gamma)$ decreases with $\tan \beta$. Hence, overall the largest rates are obtained for $\tan \beta \approx 1$. We will check directly in our study how the searches for a pseudoscalar decaying into gauge bosons affects the parameter space.

(v) We consider the most relevant indirect constraints on the parameter space in the plane $\left(m_{H^{ \pm}}, \tan \beta\right)$. These are mainly loop processes where cancellations could occur in the loops if other sources of new physics are considered. The bounds arise mainly from $B$-physics observables [50-54] and $R_{b}=\Gamma(Z \rightarrow b \bar{b}) / \Gamma(Z \rightarrow$ hadrons) [55-57]. These constraints result in a rough bound of $\tan \beta \geq 1$ for all Yukawa types. Regarding the charged Higgs mass the most relevant bound comes from $b \rightarrow s \gamma$ (type II and Y only) and is at present $m_{H^{ \pm}} \geq 570 \mathrm{GeV}[58,59]$. The same constraints force $\tan \beta \geq 2$ in type I and X. LHC run 1 has contributed with direct bounds in the $\left(m_{H^{ \pm}}, \tan \beta\right)$ plane with the process $p p \rightarrow t \bar{t}\left(H^{+} W^{-} b \bar{b}\right)[60,61]$. Finally, LEP provided the only direct bound on the charged Higgs mass of roughly $m_{H^{ \pm}} \geq 90 \mathrm{GeV}$ for all Yukawa types with $e^{+} e^{-} \rightarrow H^{+} H^{-}$assuming only $\mathrm{BR}\left(H^{ \pm} \rightarrow c s\right)+\mathrm{BR}\left(H^{ \pm} \rightarrow \tau \nu\right)+\mathrm{BR}\left(H^{ \pm} \rightarrow\right.$ $A W)=1[62]$.

\section{THE 2HDM EXTENDED BY AN UP-TYPE VECTORLIKE QUARK}

Vectorlike quarks (VLQ) appear naturally in various extension of the SM, such as some supersymetric models [63], models with extra dimensions [64], little Higgs models [65], and composite Higgs models [66]. VLQ are also well motivated by the fact that they can solve the Higgs boson mass instability resulting from large radiative corrections at high scales. In fact, a vectorlike top quark partner $(\mathrm{T})$ could play the same role as the superpartner of the top quark in supersymetric models. A particular feature of VLQ is that their left and righthanded components transform in the same way under the SM gauge group. Consequently, their mass terms are allowed in the Lagrangian without violating gauge invariance transformations.

There have been many studies on the phenomenology of the SM extended with VLQ $[10,11,67]$. Moreover, both 
ATLAS and CMS have performed several experimental searches for such new quarks. Direct searches by the ATLAS and CMS Collaborations have set lower limits on the mass of the single vectorlike $\mathrm{T}$ top partner in the range of 550-900 GeV at $8 \mathrm{TeV}$ [68-70] through one of its main decay channels: $T \rightarrow h t, W b, Z t$ since the new top is expected to couple predominantly to the third generation quarks. The above limit was improved in the $13 \mathrm{TeV}$ run. A lower limit on the mass of the T-quark was derived and found to be in the range $1170-1295 \mathrm{GeV}$ by ATLAS and CMS at $13 \mathrm{TeV}$ [71-74]. This lower limit can be lowered if the new T-quark has a non-negligible mixing with the first and second generation quarks [75].

In the present study, we consider an extension of the 2HDM by adding a vectorlike top quark (T) with charge $+\frac{2}{3}$. This extension was already studied in detail in $[12,76,77]$. Similarly to the SM, we introduce left and right components of the new top: $T_{L}^{0}, T_{R}^{0}$. The 2HDM-VLQ Lagrangian with the new top-quark $T$ is given by:

$$
\begin{aligned}
-\mathcal{L}_{Y} \supset y_{T} \overline{Q_{L}^{0}} \tilde{H}_{2} T_{R}^{0}+\lambda_{T} \overline{Q_{L}^{0}} \tilde{H}_{1} T_{R}^{0}+m_{T} \overline{T_{L}^{0}} T_{R}^{0}+\text { H.c. } \\
=y_{T}\left(\overline{t_{L}^{0}}, \overline{b_{L}^{0}}\right)\left(\begin{array}{c}
\frac{\varphi_{2}^{0}-i A}{\sqrt{2}} \\
-H^{-}
\end{array}\right) T_{R}^{0}+\lambda_{T}\left(\overline{t_{L}^{0}}, \overline{b_{L}^{0}}\right)\left(\begin{array}{c}
\frac{v+\varphi_{1}^{0}-i G^{0}}{\sqrt{2}} \\
-G^{-}
\end{array}\right) T_{R}^{0} \\
\quad+m_{T} \overline{T_{L}^{0}} T_{R}^{0}+\text { H.c., }
\end{aligned}
$$

where $\tilde{H}_{i} \equiv i \tau_{2} H_{i}^{*}$ and $Q_{L}^{0}$ is the left handed third generation quark doublets. Note that this Lagrangian is valid for all 2HDM-VLQ types because the couplings to the top are the same in all models.

After spontaneous symmetry breaking, the top quark mixes with $\mathrm{T}$ and the mass matrix of the mixing between $\left(t_{L}^{0}, T_{L}^{0}\right)$ and $\left(t_{R}^{0}, T_{R}^{0}\right)$ is given by (where we can rotate away one off-diagonal element of the mass mixing matrix).

$$
\mathcal{M}=\left(\begin{array}{cc}
\frac{y_{t} v}{\sqrt{2}} & \frac{\lambda_{T} v}{\sqrt{2}} \\
0 & m_{T}
\end{array}\right),
$$

where $y_{t}$ and $\lambda_{T}$ are the Yukawa couplings for the top quark and $\mathrm{T}$.

This matrix can be diagonalized by rotating the weak eigenstates $\left(t_{L}^{0}, T_{L}^{0}\right)$ into the mass eigenstates $\left(t_{L}, T_{L}\right)$ using a bi-unitarity transformations.

$$
\left(\begin{array}{c}
t_{L, R} \\
T_{L, R}
\end{array}\right)=\mathcal{U}_{L, R}\left(\begin{array}{c}
t_{L, R}^{0} \\
T_{L, R}^{0}
\end{array}\right),
$$

where the unitarity matrices are given by

$$
\mathcal{U}_{L, R}=\left(\begin{array}{cc}
c_{L, R} & -s_{L, R} \\
s_{L, R} & c_{L, R}
\end{array}\right)
$$

with $c_{L, R}=\cos \left(\theta_{L, R}\right)$ and $s_{L, R}=\sin \left(\theta_{L, R}\right)$. Thus the mass mixing matrix $\mathcal{M}$ is diagonalized as follows:

$$
\mathcal{U}_{L} \mathcal{M U}_{R}^{\dagger}=\mathcal{M}_{\text {diag }}=\left(\begin{array}{cc}
m_{t} & 0 \\
0 & m_{T}
\end{array}\right)
$$

or similarly

$$
\mathcal{U}_{R, L} \mathcal{M}^{\dagger} \mathcal{M U}_{R, L}^{\dagger}=\mathcal{M}_{\text {diag }}^{2} .
$$

From the fact that the off-diagonal elements of (7) vanish, one obtains the following relations for the mixing angles,

$$
\begin{aligned}
\tan \left(2 \theta_{L}\right) & =\frac{4 m_{t} m_{T}}{2 m_{T}^{2}-2 m_{t}^{2}-\lambda_{T}^{2} v^{2}}, \\
\tan \left(2 \theta_{R}\right) & =\frac{2 \sqrt{2} \lambda_{T} m_{t} v}{2 m_{T}^{2}+2 m_{t}^{2}-\lambda_{T}^{2} v^{2}} .
\end{aligned}
$$

We stress that the above mixing angles are not independent, by using Eq. (6) one can derive the following relations

$\tan \theta_{R}=\frac{m_{t}}{m_{T}} \tan \theta_{L}, \quad \frac{\lambda_{T}}{y_{t}}=\sin \theta_{L} \cos \theta_{L} \frac{m_{T}^{2}-m_{t}^{2}}{m_{t} m_{T}}$.

It is important to mention that both the interaction of the top-quark and of the $\mathrm{T}$ vector quark with the electroweak gauge bosons depend on the mixing angle $\theta_{L}$ and are given by

$$
\begin{aligned}
\mathcal{L}^{N C}= & \frac{g}{\cos \theta_{W}} Z_{\mu}\left(\left[\frac{\cos \theta_{L}^{2}}{2}-\frac{2}{3} \sin ^{2} \theta_{W}\right] \overline{t_{L}} \gamma^{\mu} t_{L}\right. \\
& +\left[\frac{\sin \theta_{L}^{2}}{2}-\frac{2}{3} \sin ^{2} \theta_{W}\right] \overline{T_{L}} \gamma^{\mu} T_{L} \\
& \left.+\frac{\sin \theta_{L} \cos \theta_{L}}{2}\left[\overline{T_{L}} \gamma^{\mu} t_{L}+\text { H.c }\right]\right) . \\
\mathcal{L}^{C C}= & \frac{g}{\sqrt{2}}\left(\cos \theta_{L} \overline{t_{L}}+\sin \theta_{L} \overline{T_{L}}\right) b \gamma^{\mu} W_{\mu}^{+}+\text {H.c.. }
\end{aligned}
$$

After EWSB one can derive the following Yukawa couplings of the $C P$-odd Higgs A to both $t$ and $T$

$$
\begin{aligned}
A t \bar{t} & =-i\left(\frac{c_{L} c_{R}}{\tan \beta}-\frac{c_{L} s_{R} y_{T}}{y_{t}}\right), \\
A T \bar{T} & =-i\left(\frac{s_{L} s_{R}}{\tan \beta}+\frac{s_{L} c_{R} y_{T}}{y_{t}}\right), \\
A \bar{t}_{L} T_{R} & =-i\left(\frac{c_{L} s_{R}}{\tan \beta}+\frac{c_{L} c_{R} y_{T}}{y_{t}}\right), \\
A t_{R} \bar{T}_{L} & =-i\left(\frac{s_{L} c_{R}}{\tan \beta}-\frac{s_{L} s_{R} y_{T}}{y_{t}}\right) .
\end{aligned}
$$

After this brief review of the couplings of the heavy top to gauge bosons and Higgs bosons, we list hereafter the most important theoretical and phenomenological constraints on the parameters of the model. From the 
theoretical side, the scalar sector of $2 \mathrm{HDM}+\mathrm{T}$ is subject to the same unitarity and vacuum stability constraints as the usual 2HDM [32,38-40]. On the other hand, $y_{T}$ is also constrained from unitarity to be less than $4 \pi$ while $\lambda_{T}$ is a derived quantity [see Eq. (9)].

(i) The above interactions of $t_{R}$ and $T_{R}$ with the $Z$ and $W$ bosons in the $2 \mathrm{HDM}+\mathrm{T}$ are the same as those in the SM. Since the new top will contribute to gauge bosons self energies, the mixing angle $\theta_{L}$ can be constrained from electroweak observables such as $\mathrm{S}$ and $\mathrm{T}$ parameters [12]. It has been shown in [12] that $\sin \theta_{L}<0.2$ (resp 0.12) for $m_{T}=400 \mathrm{GeV}$ (resp $m_{T}=1 \mathrm{TeV}$.)

(ii) The interaction of the heavy top with charged Higgs and bottom quark can affect the rate of $\mathrm{BR}(b \rightarrow s \gamma)$ [12]. This can be translated into constraints on the charged Higgs mass and/or mixing angle between heavy top and top quark. In the case where we assume that $\sin \theta_{L} \approx V_{T b}$, it has been shown in [12] that in 2HDM-II, $V_{T b}$ must be smaller than 0.03 for $m_{T}>600 \mathrm{GeV}$. This limit can be weakened for light $m_{T}$.

(iii) Regarding compatibility with the couplings of the $h_{125}$ Higgs to the remaining SM particles, since we are working in the alignment limit all couplings are SM-like except the couplings to top quarks for which we choose a small mixing angle to force compatibility with the measured Higgs couplings. Regarding the searches for heavy scalars, the situation is exactly the same as for the 2HDM. So again we use the limits derived for the scalar decay to fermions and study the effect of the decay to gauge bosons in the parameter space of the model.

Moreover, since the new heavy top couples to the all Higgs bosons, the decay patterns $T \rightarrow b W, h t, Z t$ will be modified. It is well known that in the SM extended with a heavy top the values of $\operatorname{BR}(T \rightarrow b W), \operatorname{BR}(T \rightarrow h t)$ and $\mathrm{BR}(T \rightarrow Z t)$ are, respectively, $50 \%, 25 \%$, and $25 \%$. In the $2 \mathrm{HDM}$ with the presence of new Higgs bosons $A, H$ and $H^{ \pm}$, the above pattens are modified $[12,77]$ and the limits obtained by ATLAS and CMS have to be reinterpreted in the framework of the $2 \mathrm{HDM}+\mathrm{T}$.

\section{RESULTS FOR THE 2HDM}

In this section, we present the results for the $p p \rightarrow A \rightarrow$ $V V$ production rates, where $V=\gamma, Z, W$, evaluated in the narrow width approximation. The pseudoscalar production cross section was calculated using SUSHI [78] at NNLO and it includes gluon and $b \bar{b}$ fusion. The branching ratios were calculated using the HDECAY [79-81] version for the 2HDM [82]. The widths for the pseudoscalar decays into vector bosons are loop induced and were calculated with the packages FeYnARTs [83], FormCalC [84] and LoopTools $[84,85]$ for loop integrals evaluation. The loop calculation is performed in the 't Hooft-Feynman gauge using dimensional regularization. At the one-loop level, only fermionic loops contribute to $A \rightarrow V V$. The reason is that in the bosonic sector, the electroweak theory conserves $C P$ while after adding fermions $C P$ is no longer conserved. Therefore, there is no contribution to $A \rightarrow V V$ from the SM bosons [9], or from the spin zero scalars but only from the fermions. Analytical and numerical check of UV finiteness have been performed.

As the measurements of the Higgs couplings to the other SM particles become increasingly precise, the $2 \mathrm{HDM}$ approaches more and more the alignment limit where $\sin (\beta-\alpha) \approx 1$ if $h$, the lightest $C P$-even scalar, is the SM-like Higgs boson. Considering the lower limit on the charged Higgs mass (about $100 \mathrm{GeV}$ for type I and X and $600 \mathrm{GeV}$ for type II and Y) and that $m_{h}=125 \mathrm{GeV}$, the decay $A \rightarrow h Z$ is kinematically allowed for a pseudoscalar mass of more than about $200 \mathrm{GeV}$ for all types while $A \rightarrow H^{ \pm} W^{\mp}$ would be allowed for a pseudoscalar mass of about 200 (700) GeV for type I and X (type II and Y). In this study, we will choose the heavy Higgs masses, $m_{H^{ \pm}}$ and $m_{H}$, to be equal and such that the decay $A \rightarrow H^{ \pm} W^{\mp}$ is kinematically disallowed, which is already the case for models type II and Y due to the combined experimental and theoretical limits. The decay $A \rightarrow h Z$ is exactly zero in the alignment limit. By extending this condition to all models we are being conservative in the chances for the detection of a pseudoscalar in $V V$ final states. In fact, as more decays of the pseudoscalar are kinematically available, the chances of detecting a pseudoscalar in $V V$ final states become smaller. The range of variation of the pseudoscalar mass is chosen to be in the range $50 \mathrm{GeV}<m_{A}<600 \mathrm{GeV}$ for $A \rightarrow \gamma \gamma$ and $2 m_{V}<m_{A}<600 \mathrm{GeV}$ for $A \rightarrow V V, V=W$, $Z$. In this mass range, the main decay channels of the pseudoscalar are $A \rightarrow b \bar{b}, A \rightarrow \tau^{+} \tau^{-}$and $A \rightarrow \gamma \gamma$ (also $A \rightarrow Z Z\left(W^{+} W^{-}\right)$are possible but at much lower rate as will be discussed later). Clearly, the $A \rightarrow \gamma \gamma$ rate is at least 2 orders of magnitude below the tree-level decays so one could ask how important this decay really is. Taking into account the analysis performed for the $125 \mathrm{GeV}$ SM Higgs where the ratio

$$
\mathrm{BR}(h \rightarrow \gamma \gamma) / \mathrm{BR}\left(h \rightarrow \tau^{+} \tau^{-}\right) \approx 0.0362
$$

holds and still the two photons channel was the first one to be measured, we can expect that for a factor of about 100 the $A \rightarrow \gamma \gamma$ channel will still be competitive due to sharp resolution in the di-photon invariant mass achievable by the CMS and ATLAS detectors. The above number will be used a rough guide to what can be expected from future analyses.

In Fig. 1, we show $\operatorname{BR}(A \rightarrow \gamma \gamma) / \operatorname{BR}(A \rightarrow \tau \tau)$ as a function of $m_{A}$ for $\tan \beta=1$ and $\tan \beta=10$ in type I and type II. Also shown is the SM line for the same ratio. In type I, the two lines for $\tan \beta=1$ and $\tan \beta=10$ overlap because $A \rightarrow \gamma \gamma$ is mediated only through fermionic loops 

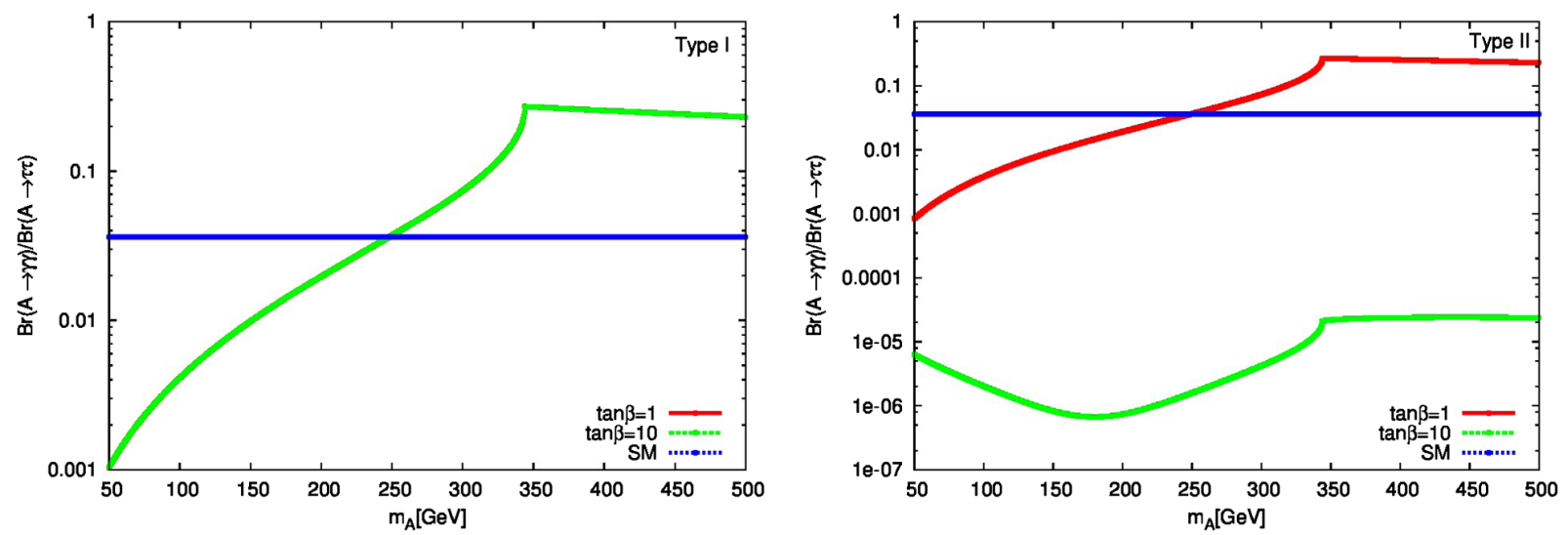

FIG. 1. $\operatorname{BR}(A \rightarrow \gamma \gamma) / \operatorname{BR}(A \rightarrow \tau \tau)$ as a function of $m_{A}$ for $\tan \beta=1$ and $\tan \beta=10$ in type I and type II.

and the $A f \bar{f} \propto 1 / \tan \beta$ coupling factorizes out and cancels with the same factor coming from $A \rightarrow \tau \tau$. In type I, the SM line is crossed for $m_{A} \approx 250 \mathrm{GeV}$ independently of $\tan \beta$. However, since both the production cross section and the luminosity will be much higher during Run 2 it is to be expected that at very high luminosity all values of the pseudoscalar mass will be probed by the end of the LHC $14 \mathrm{TeV}$ run in the low $\tan \beta$ region. The same behavior is seen in type II for low $\tan \beta$. As $\tan \beta$ increases it will become increasingly harder to detect a pseudoscalar in the two-photons final state. It should be mentioned that the width of $A \rightarrow V V$, whatever the final state is, is controlled by the top-quark loop. This loop is always proportional to $1 / \tan ^{2} \beta$ and therefore the width is the same for all Yukawa versions of the model except for very large values of $\tan \beta$ in type II and Y. The behavior we see in the right panel of Fig. 1 is due to the width of $A \rightarrow \tau \tau$ that increases with $\tan \beta$ in type II.

\section{A. $A \rightarrow \gamma \gamma$}

We now move to the detailed study of the production rates of a pseudoscalar decaying into two photons at the LHC at $8 \mathrm{TeV}$ and $14 \mathrm{TeV}$. In Fig. 2, we plot the pseudoscalar production cross section multiplied by the branching ratio $\operatorname{BR}(A \rightarrow \gamma \gamma)$ for all four Yukawa types as a function of $m_{A}$. The remaining masses are fixed at $m_{h}=125 \mathrm{GeV}$ and $m_{H^{+}}=m_{H}=600 \mathrm{GeV}$. Regarding the angles we take the exact alignment limit $\sin (\beta-\alpha)=1$ and we scan over $\tan \beta$ from 1 to 40 , except for type II, where the scans stops at 10 . As previously discussed, the largest rates are for small $\tan \beta$ and therefore the upper bound is not relevant for the discussion. In the same plots, we show the limits obtained by ATLAS [86] and CMS [87,88] after Run 1 . The situation is similar for all models: only a small region of the parameter space where $\tan \beta$ is small is excluded with this search. If we move to the large $\tan \beta$ and/or to large pseudoscalar mass the number of events becomes negligible. The increase in cross section in type II and $\mathrm{Y}$ due to $b b$ production (the $b b A$ coupling is proportional to $\tan \beta$ ) is not enough to compensate the decrease in branching ratio. Therefore the number of events will be small even for large $\tan \beta$ except for the region of very small pseudoscalar mass.

In Fig. 3, we again show a scan in the type I and type II models for $14 \mathrm{TeV}$ and with the exact same conditions of the previous Fig. 2. The pseudoscalar production cross section increases from a factor of about 2 for $m_{A}=50 \mathrm{GeV}$ to a factor of about 4 for $m_{A}=600 \mathrm{GeV}$. An extra factor coming from the $b b$ initiated process will further increase the production cross section for type II and Y especially for pseudoscalar masses above the $t \bar{t}$ threshold. Still this factor is quite small: taking for instance $m_{A}=500 \mathrm{GeV}$ the cross section increases by about $20 \%$ for $\tan \beta=40$. Regarding the luminosity, which is expected to be about $300 \mathrm{fb}^{-1}$ at the end of run 2 , when compared to the $8 \mathrm{TeV}$ run where the total luminosity collected was about $30 \mathrm{fb}^{-1}$, there is an approximate tenfold increase. Overall a factor below 20 can be foreseen for the low mass region. Therefore, it is clear that it will be hard to probe this channel above about $\tan \beta=10$ (and probably less) by the end of Run 2 .

$$
\text { B. } A \rightarrow Z Z\left(W^{+} W^{-}\right)
$$

The decay rate of a pseudoscalar to massive gauge bosons in the 2HDM is extremely small. The reason is clear: the decays of pseudoscalar bosons to massive gauge bosons can only occur at the loop level and massive gauge bosons are heavy. In fact, $\Gamma(A \rightarrow Z Z)$ is always smaller than $10^{-5} \mathrm{GeV}$ below the $t \bar{t}$ threshold and smaller than $10^{-4} \mathrm{GeV}$ above the same threshold, independently of the Yukawa version of the model. It is therefore clear that these are not competitive channels when compared to the ones with two fermions or even two photons final states. Since we are considering pseudoscalar production via fermion loops or $b \bar{b}$ initiated processes, a pseudoscalar decaying to two massive gauge bosons is expected to be observed well after being detected in some fermion final state $\left(\tau^{+} \tau^{-}, b \bar{b}\right.$ or $t \bar{t}$ ) or in $\gamma \gamma$. Similarly to the two-photon final state, also here we can use exclusion bounds from searches for a scalar 

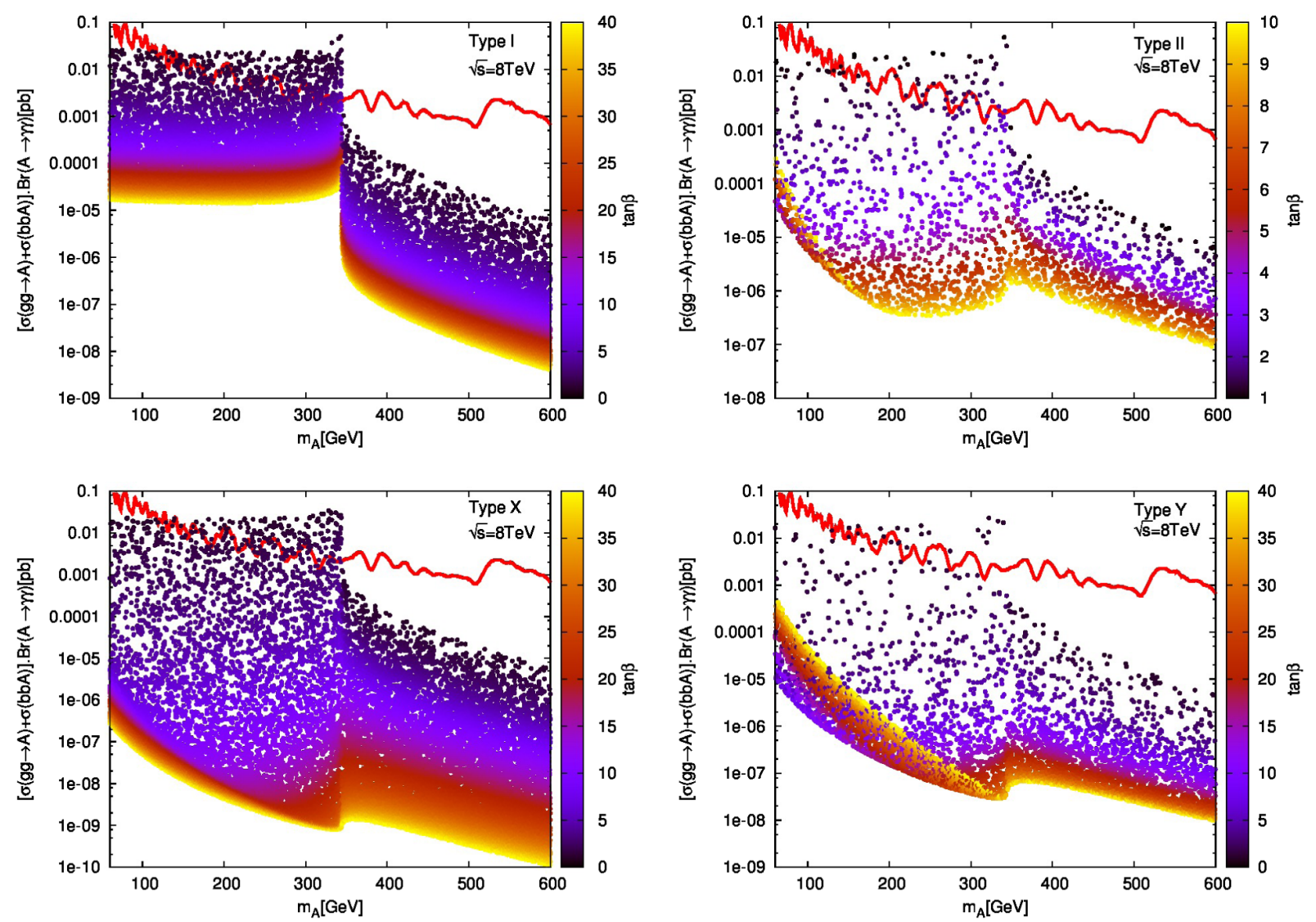

FIG. 2. $[\sigma(g g \rightarrow A)+\sigma(b b A)] \operatorname{BR}(A \rightarrow \gamma \gamma)$ at $\sqrt{s}=8 \mathrm{TeV}$ as a function of $m_{A}$ with $m_{H^{+}}=m_{H}=600 \mathrm{GeV}$. The values of tan $\beta$ are color coded as indicated on the right of the plots. Also shown is the exclusion line from ATLAS (see text).

decaying to either two $Z$ bosons or to $W^{+} W^{-}$performed by the ATLAS $[89,90]$ and CMS [91] collaborations. In Fig. 4, we present the pseudoscalar production cross section multiplied by the branching ratio $A \rightarrow Z Z$ (top) and $A \rightarrow$ $W^{+} W^{-}$(bottom) for type I (left) and type II (right). We also present the best experimental upper exclusion bound for these channels $[89,90]$. It is clear that the experimental bounds are still about 1 order of magnitude away from the points with the largest rates in the scan. Moreover, also for these final states only points below the $t \bar{t}$ threshold and in the low $\tan \beta$ region have some chances of being probed at the next LHC run. As previously discussed for the two photon final state there is an overall factor of about 20 for the low mass region when considering both the increase in cross section and in luminosity. However, Fig. 4 clearly shows that, even if the results are better by 2 orders of
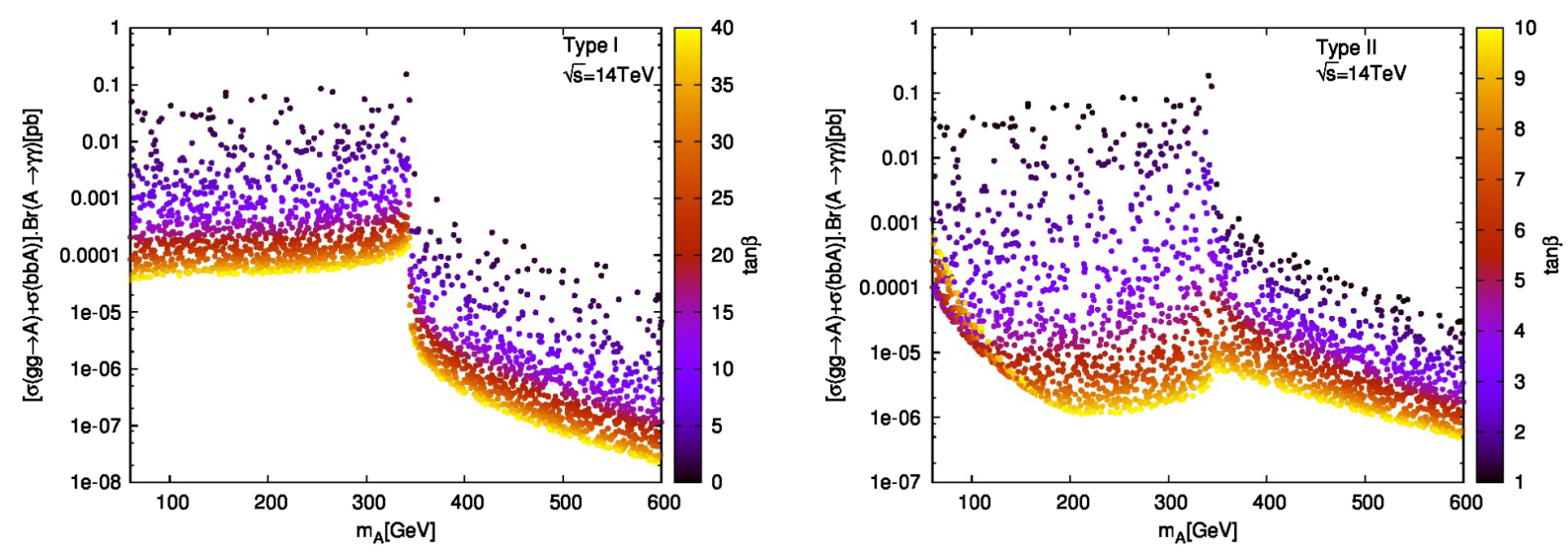

FIG. 3. $[\sigma(g g \rightarrow A)+\sigma(b b A)] \mathrm{BR}(A \rightarrow \gamma \gamma)$ at $\sqrt{s}=14 \mathrm{TeV}$ as a function of $m_{A}$ with $m_{H^{+}}=m_{H}=600 \mathrm{GeV}$. The values of tan $\beta$ are color coded as indicated on the right of the plots. 

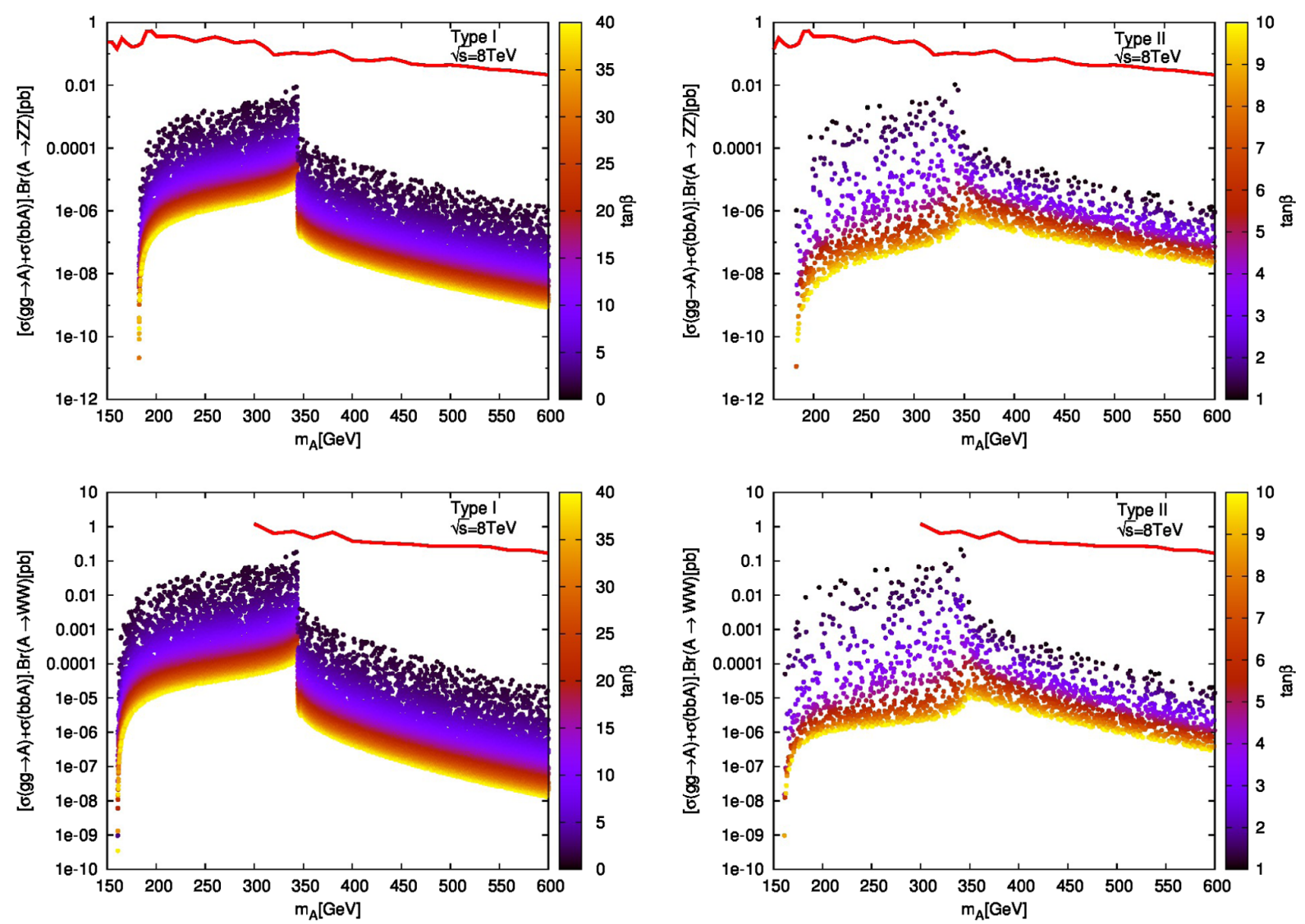

FIG. 4. $[\sigma(g g \rightarrow A)+\sigma(b b A)] \mathrm{BR}(A \rightarrow Z Z)$ (top) and $[\sigma(g g \rightarrow A)+\sigma(b b A)] \mathrm{BR}(A \rightarrow W W)$ (bottom) at $\sqrt{s}=8 \mathrm{TeV}$ as a function of $m_{A}$ with $m_{H^{+}}=m_{H}=600 \mathrm{GeV}$. The values of $\tan \beta$ are color coded as indicated on the right of the plots.

magnitude, we will barely start to probe a few scenarios in the low $\tan \beta$ region. In Fig. 5, we present $[\sigma(g g \rightarrow A)+$ $\sigma(b b A)] \mathrm{BR}(A \rightarrow Z Z)$ at $\sqrt{s}=14 \mathrm{TeV}$ as a function of $m_{A}$ with $m_{H^{+}}=m_{H}=600 \mathrm{GeV}$. As discussed, both for type I and type II, there is an increase in the maximum values of the rates but still well below the experimental result line and an increase in more than 1 order of magnitude is needed to start probing the largest values of the rates.

\section{RESLUTS FOR THE 2HDM+T}

Before we present our results for $2 \mathrm{HDM}$ with a vectorlike top, we first show in Fig. 6 the allowed range for $y_{T}, \tan \beta$, the mixing angles $\alpha$ and $\sin \theta_{L}$ for a fixed $m_{T}=1 \mathrm{TeV}$. From the left plot of Fig. 6, one can see that $\left|y_{T}\right| \leq 15$ is allowed and that the mixing angle $\sin \theta_{L}$ should be less than about 0.2 for any value of
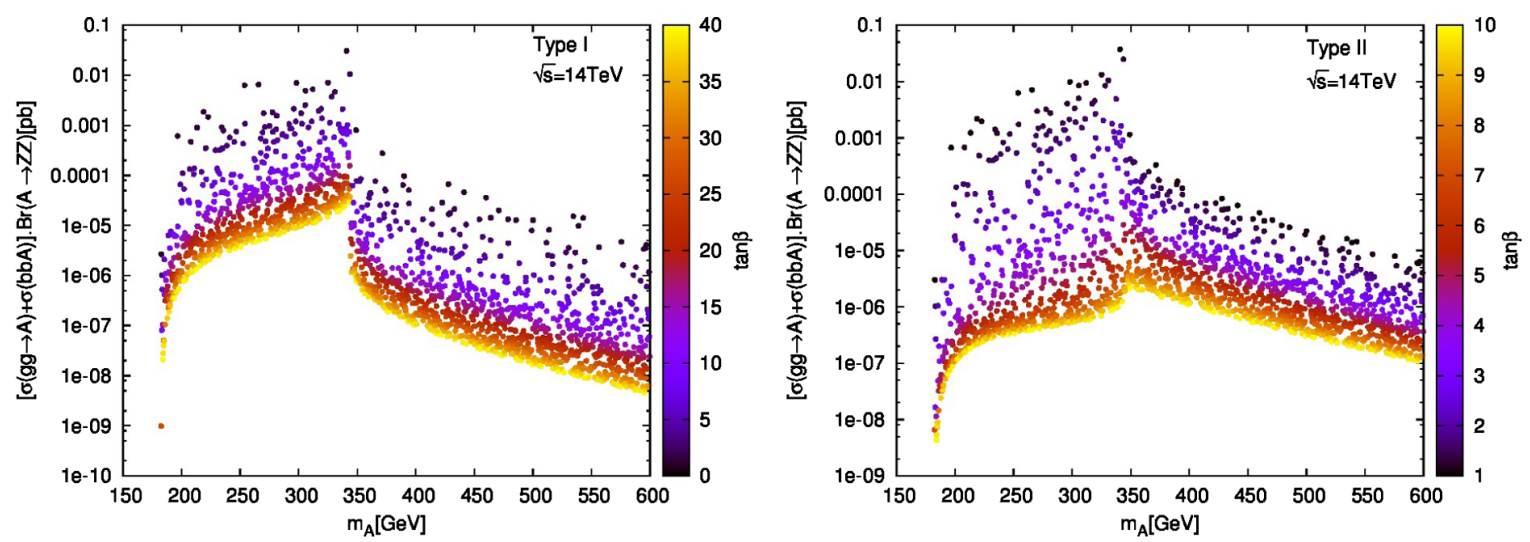

FIG. 5. $[\sigma(g g \rightarrow A)+\sigma(b b A)] \mathrm{BR}(A \rightarrow Z Z)$ at $\sqrt{s}=14 \mathrm{TeV}$ as a function of $m_{A}$ with $m_{H^{+}}=m_{H}=600 \mathrm{GeV}$. The values of tan $\beta$ are color coded as indicated on the right of the plots. 

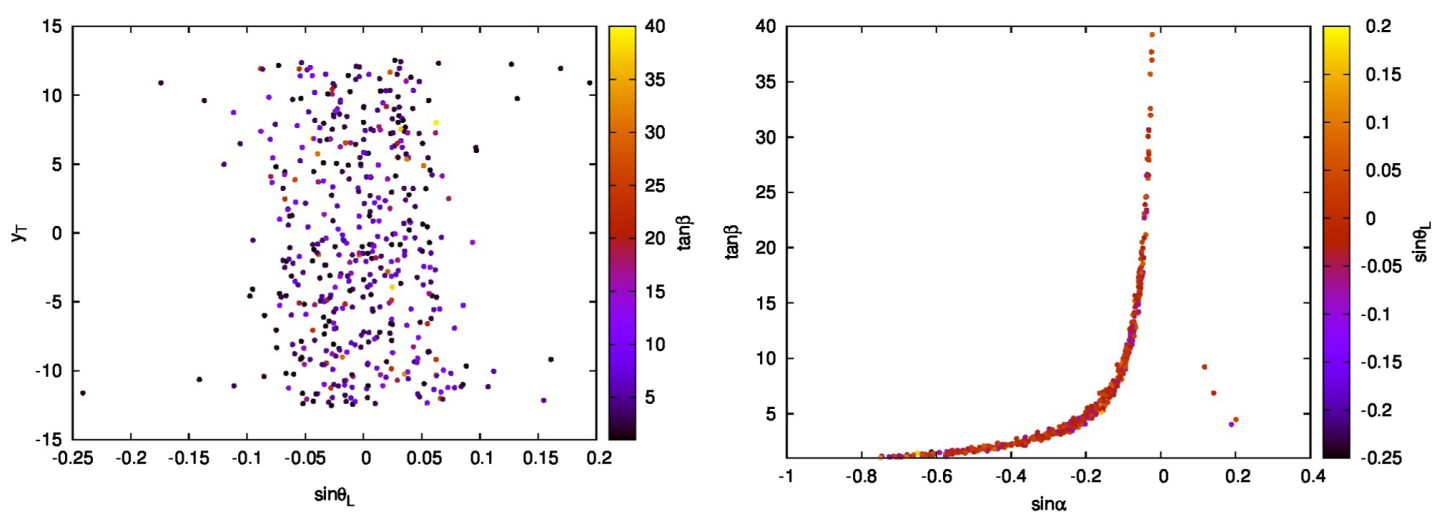

FIG. 6. Results of the scan described in the text in the $\left(\sin \theta_{L}, y_{T}\right)$ plane (left) and in the ( $\left.\sin \alpha, \tan \beta\right)$ plane (right), after imposing the most relevant theoretical and experimental constraints as previously described in detail $\left(m_{T}=1 \mathrm{TeV}\right)$.

$1 \leq \tan \beta \leq 40$. In the right panel, we show $\tan \beta$ as a function of $\sin \alpha$ where we can see a very similar behavior to the 2HDM for the same variables, indicating that we are again very close to alignment except for a few points in the region where $\sin \alpha>0$ where the coupling to b-quarks change sign, known as the wrong sign limit $[92,93]$.

Extending the 2HDM to include a vectorlike top quark, results in an enhancement both in the pseudoscalar production cross section and in its decay width into $\gamma \gamma$, $Z Z$ and $W^{+} W^{-}$. Hence, even with all constraints taken into account, in the case of large mixing between the top quark and new top $\mathrm{T}$, the production rates of loop-induced processes can be several orders of magnitude above the $2 \mathrm{HDM}$ ones. In order to see the effects of the new top in the production rates of the gauge bosons, we plot in Fig. 7 the rate $[\sigma(g g \rightarrow A)+\sigma(b b A)] \operatorname{BR}(A \rightarrow \gamma \gamma, Z Z, W W)$ at $\sqrt{s}=8 \mathrm{TeV}$ as a function of $m_{A}$ (and $\tan \beta$ ), where we chose $m_{T}=1 \mathrm{TeV}, y_{T}=10$ and $\sin \left(\theta_{L}\right)=0.12$. One can see from this plot that the effect of the new top on the production and the decay of the $C P$-odd Higgs is a significant enhancement in both the production cross section by gluon fusion and in the decays $A \rightarrow \gamma \gamma, A \rightarrow$ $Z Z$ and $A \rightarrow W^{+} W^{-}$when compared to the ones in the 2HDM (note that the Higgs production in bottom-quark annihilation is not altered by the new top).

As is clear from Fig. 7, the largest enhancement in the rates is again obtained for low $\tan \beta$, as in the 2HDM. Like in the 2HDM, this behavior can be understood by looking at the couplings given in Eq. (12), valid both for $2 \mathrm{HDM}+\mathrm{T}$ types I and II, which show that all couplings contain a term proportional to $1 / \tan \beta$. Another source of enhancement relative to the $2 \mathrm{HDM}$ is the choice of large Yukawa $y_{T}$, which is still well below the perturbativity limit of $4 \pi$, as well as large mixing $\sin \theta_{L}$. Also shown in the plots are the exclusion lines from the experiments at CERN. In the upper plots, we can see the diphoton exclusion line from ATLAS. This plot is presented to show a very interesting result: in a type I $2 \mathrm{HDM}+\mathrm{T}$, a pseudoscalar with $m_{A}<$ $2 m_{t} \approx 350 \mathrm{GeV}$ and large mixing $\sin \theta_{L} \approx 0.12-0.06$ is excluded for any value of $\tan \beta$. This means that the twophoton final state search is important to further constrain models with vectorlike quarks. One should note however that away from $t \bar{t}$ threshold the exclusion is valid only for rather small $\tan \beta$. In the type II $2 \mathrm{HDM}+\mathrm{T}$, one can see that for $m_{A} \leq 350 \mathrm{GeV}$ small $\tan \beta$ is excluded from diphoton events. We note that there is no exclusion for small $\sin \theta_{L} \leq$ 0.01 both for type I and type II. In the middle and lower panels of Fig. 7, we present the rates for $\sigma(p p \rightarrow A) \times$ $\mathrm{BR}(A \rightarrow V V)$ for $\mathrm{V}=Z$ or $\mathrm{W}$ together with the exclusion line from ATLAS. In both cases, the total rates are still about 1 order of magnitude smaller than the exclusion line. We note that there is a slight enhancement in the rate $\sigma(p p \rightarrow A) \times \mathrm{BR}(A \rightarrow Z Z)$ with respect to the $2 \mathrm{HDM}$ case, due to the extra loop contribution. Still depending on the parameters chosen, the rate $\sigma(p p \rightarrow A) \times \mathrm{BR}(A \rightarrow V V)$ for $V=Z, W$ may also be suppressed compared to the 2HDM.

\section{AT A 100 TEV PP COLLIDER}

In the quest for new physics, there is a consensus among the community of particle physicists in favor of the construction a high-energy machine with $100 \mathrm{TeV}$ center of mass energy. One question that has been raised about a future $100 \mathrm{TeV} p p$ collider is what is the luminosity needed to address the physics that is not within the reach of the LHC, even at high luminosity. In [94], several physics scenarios were analyzed and a luminosity of about $10-20 \mathrm{ab}^{-1}$ was shown to be a good compromise in extending the discovery reach for new phenomena relative to the high luminosity LHC.

The production cross sections $p p \rightarrow A$ at a $100 \mathrm{TeV}$ collider is increased relative to the $8 \mathrm{TeV}$ LHC from a factor of about 20 for $m_{A}=50 \mathrm{GeV}$ to about 220 for $m_{A}=600 \mathrm{GeV}$. This behavior is shown in the left panel of Fig. 8 where the ratio of the cross sections for $100 \mathrm{TeV}$ and for $8 \mathrm{TeV} \sigma(p p \rightarrow A)_{100 \mathrm{TeV}} / \sigma(p p \rightarrow A)_{8 \mathrm{TeV}}$ is shown as a function of the pseudoscalar mass for type I and two 

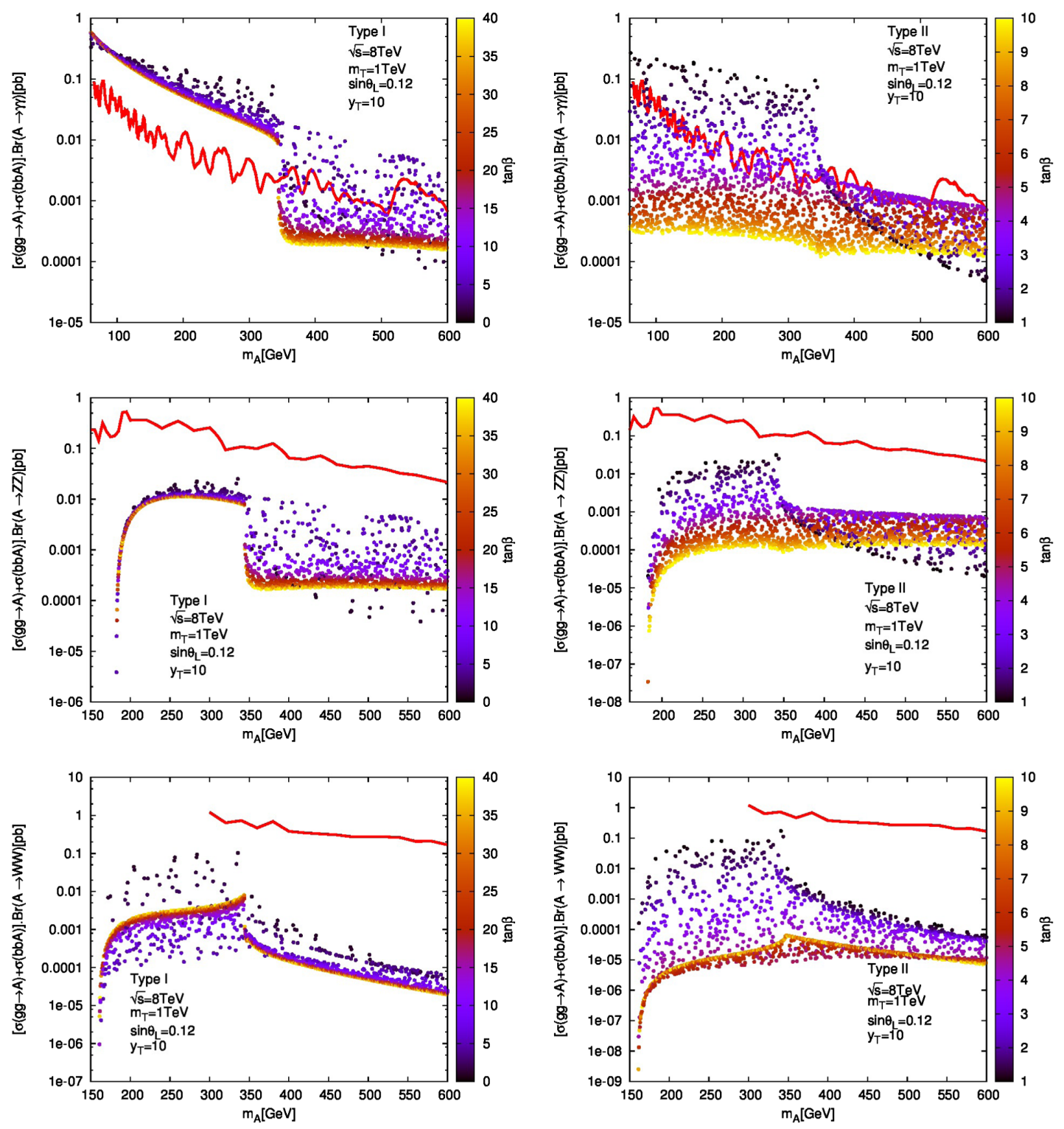

FIG. 7. Scatter plots of $[\sigma(g g \rightarrow A)+\sigma(b b A)] \mathrm{BR}(A \rightarrow \gamma \gamma, Z Z, W W)$ at $\sqrt{s}=8 \mathrm{TeV}$ in the $2 \mathrm{HDM}+\mathrm{T}$ as a function of $m_{A}$ where $m_{H^{+}}=m_{H^{0}}=600 \mathrm{GeV}, \sin \left(\theta_{L}\right)=0.12, m_{T}=1 \mathrm{TeV}$ and $y_{T}=10$, the values of $\tan \beta$ are color coded as indicated on the right of the plots.

values of $\tan \beta, 1$ and 10. The plots for all other Yukawa types show exactly the same behavior as the one for type I for $\tan \beta=1$ and for large $\tan \beta$ the contribution of the $b b$ initiated process slightly changes this ratio with no meaningful changes in the conclusions.

As previously discussed, so far analyses were only performed for $8 \mathrm{TeV}$ with a total luminosity of about $30 \mathrm{fb}^{-1}$. Therefore, in the low mass region the cross section is increased by a factor of 20 while the luminosity is incremented by about 1000 . Overall, an improvement of at least 4 orders of magnitude is expected. The increase is more significant for higher masses but the branching ratios are smaller. Furthermore, the ratio of the cross sections is almost independent of $\tan \beta$ and of the Yukawa type.
Considering Fig. 2, it is clear that most of the parameter space will be probed in the case of $p p \rightarrow A \rightarrow \gamma \gamma$ for the $2 \mathrm{HDM}$, and for any Yukawa type. However, when examining Fig. 4 for the case of the decays into massive gauge bosons, we see that only a small portion of the parameter space will be probed, mainly for low $\tan \beta$ and for pseudoscalar mass below the $t \bar{t}$ threshold. The same is true for the $W^{+} W^{-}$final state.

Finally, in Fig. 9, we present scatter plots for $[\sigma(g g \rightarrow$ $A)+\sigma(b b A)] \operatorname{BR}(A \rightarrow \gamma \gamma, Z Z, W W)$ for $\sqrt{s}=100 \mathrm{TeV}$ in the 2HDM+T as a function of $m_{A}$ where $m_{H^{+}}=m_{H^{0}}=$ $600 \mathrm{GeV}, \sin \left(\theta_{L}\right)=0.12, m_{T}=1 \mathrm{TeV}$ and $y_{T}=10$. The left plots are for type I and the right plots are for type II. In the case of the $A \rightarrow \gamma \gamma$ part of the parameter space, for the 

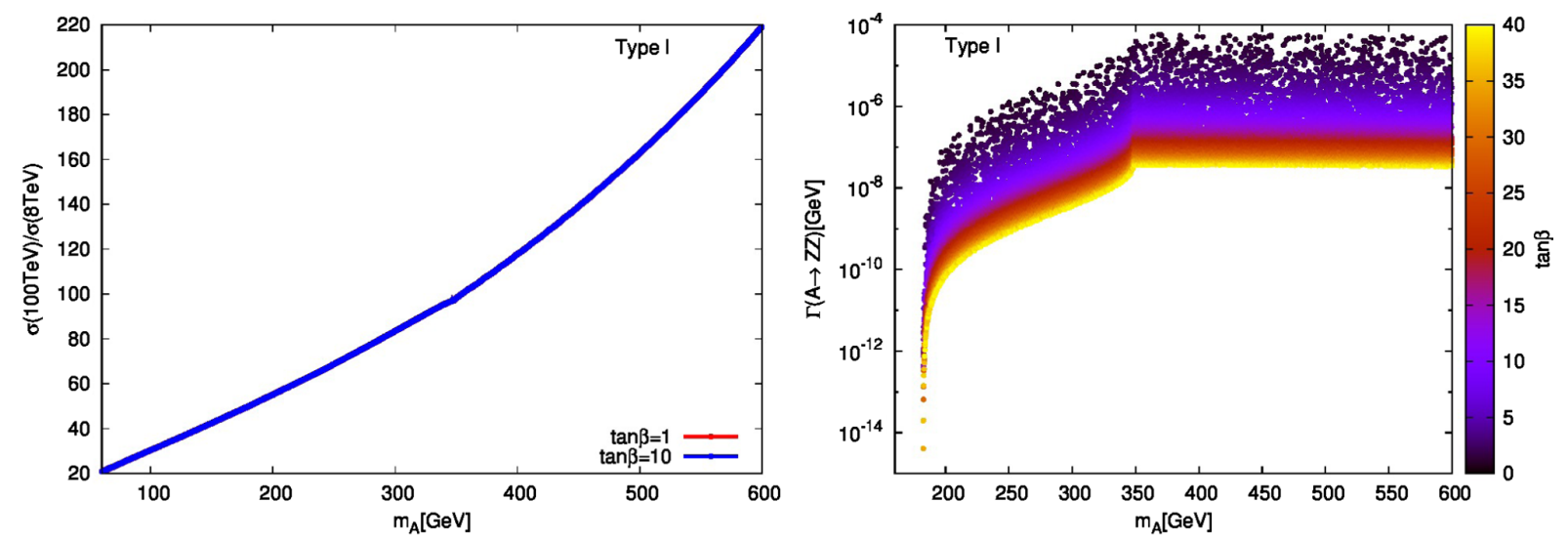

FIG. 8. Left panel: ratio of cross section $\sigma(p p \rightarrow A)_{100 \mathrm{TeV}} / \sigma(p p \rightarrow A)_{8 \mathrm{TeV}}$ as a function of $m_{A}$ for $\tan \beta=1$ and tan $\beta=10$; Right panel: partial width $\Gamma(A \rightarrow Z Z)$ as a function of $m_{A}$ for type I. The values of $\tan \beta$ are color coded as indicated on the right of the plot.
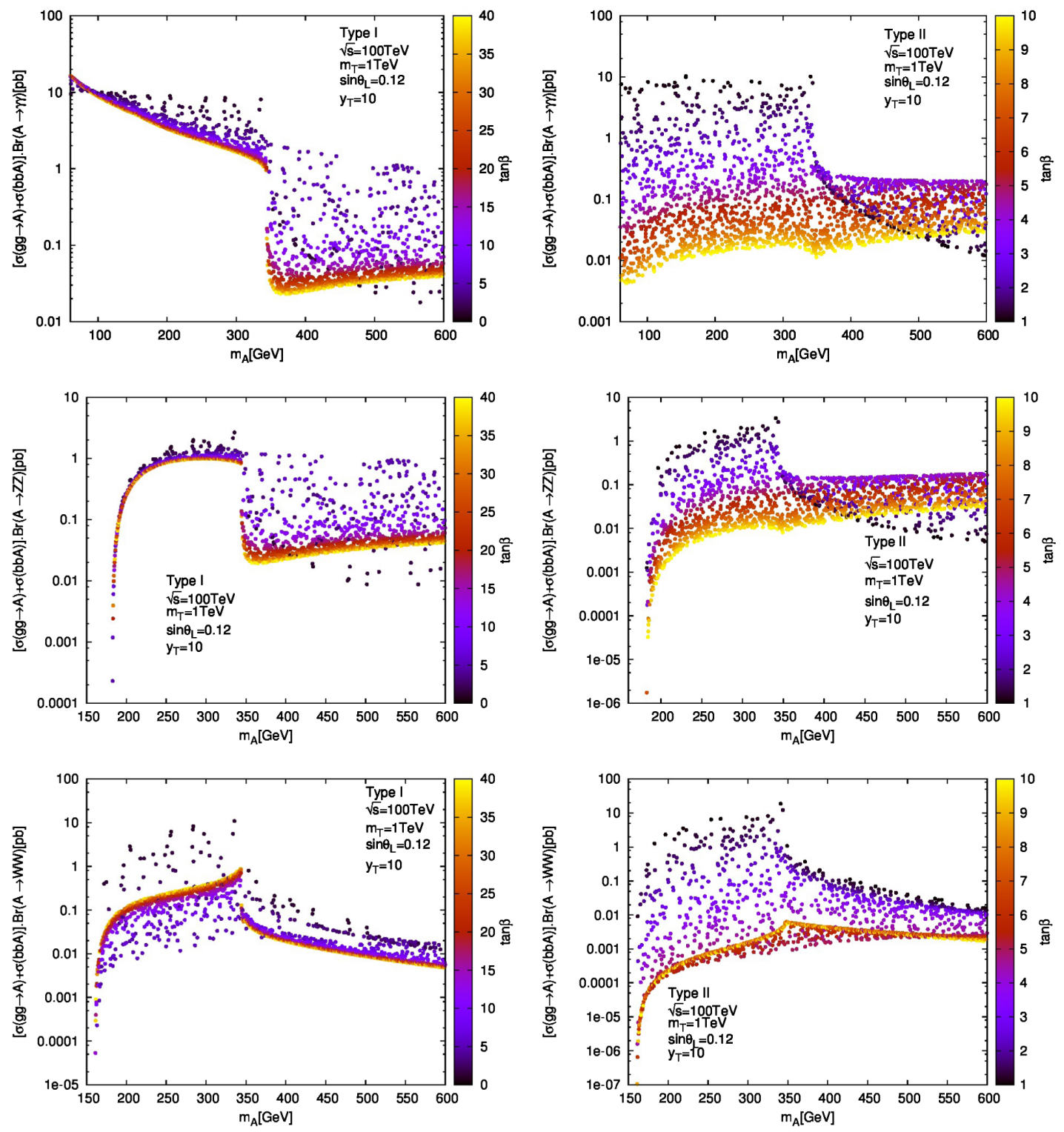

FIG. 9. Scatter plots for $[\sigma(g g \rightarrow A)+\sigma(b b A)] \mathrm{BR}(A \rightarrow \gamma \gamma, Z Z, W W)$ for $\sqrt{s}=100 \mathrm{TeV}$ in the $2 \mathrm{HDM}+\mathrm{T}$ as a function of $m_{A}$ where $m_{H^{+}}=m_{H^{0}}=600 \mathrm{GeV}, \sin \left(\theta_{L}\right)=0.12, m_{T}=1 \mathrm{TeV}$ and $y_{T}=10$, the values of $\tan \beta$ are color coded as indicated on the right of the plots. 

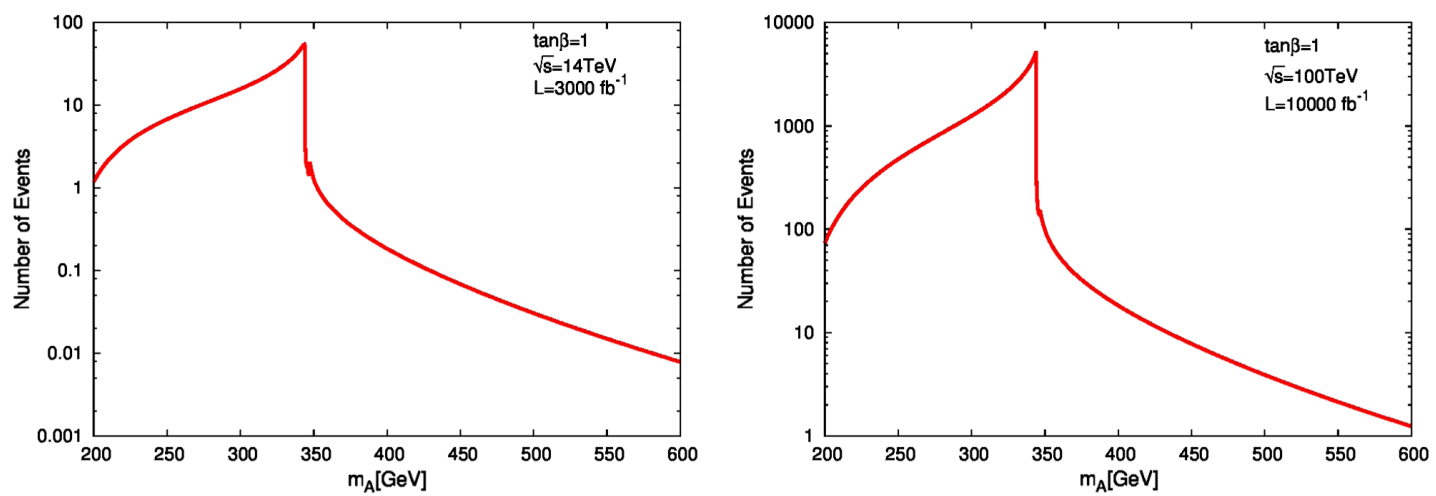

FIG. 10. Number of events obtained at the $14 \mathrm{TeV}$ LHC with a total integrated luminosity of $3000 \mathrm{fb}^{-1}$ (left) and at a $100 \mathrm{TeV}$ machine with $10 \mathrm{ab}^{-1}$ integrated luminosity for a $2 \mathrm{HDM}$ of any type (as $\tan \beta=1$ ). We assume that the efficiency factor is $\epsilon \approx 0.35$.

low mass region is already excluded for the parameters shown. For the same set of parameters almost all values of $m_{A}$ and $\tan \beta$ are within the reach of a $100 \mathrm{TeV}$ colliders. However, as $y_{T}$ decreases, the model will resemble the $2 \mathrm{HDM}$ and therefore as previously discussed only the low $\tan \beta$ region will have some chances to be probed.

In order to roughly quantify the sensitivity for the $Z Z$ and $W^{+} W^{-}$final states (where our main interest is focused), we will perform some rough estimates regarding the observability of the $C P$-odd scalar decaying to two $Z$ bosons in the four-lepton channel. Let us start by computing an upper limit of the number of events obtained for the $2 \mathrm{HDM}$ (by choosing $\tan \beta=1$ ) taking into account the efficiency factor for the four lepton channel search. As discussed in [9], for the $4 l$ channel, the only significant background is $q q, g g \rightarrow Z Z \rightarrow 4 l$, which is fully determined by the detector resolution in the 41 channel [95]. For the efficiency factor for this channel we use the number given in [95], which is $\epsilon \approx 0.35$. The number of 41 events that one would then obtain at a hadron collider is calculated as follows

$$
\begin{aligned}
N_{\text {events }}= & \sigma\left(p p \rightarrow A^{0}\right) \times \operatorname{BR}\left(A^{0} \rightarrow Z Z\right) \\
& \times(\operatorname{BR}(Z \rightarrow 2 l))^{2} \times \mathcal{L} \times \epsilon
\end{aligned}
$$

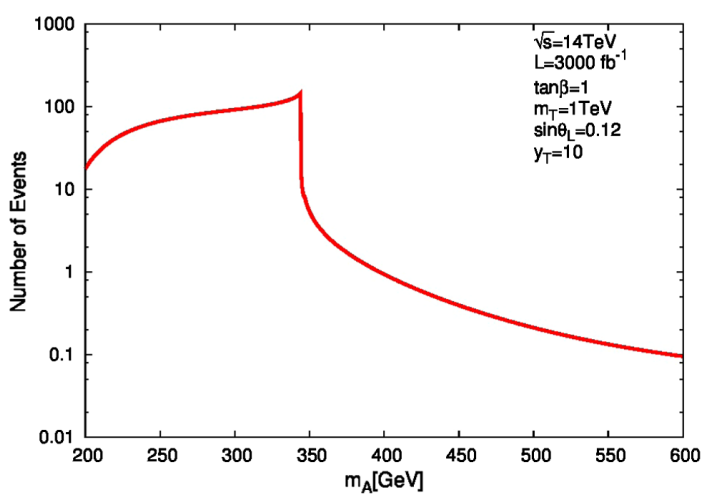

where we will use the predicted collected luminosity at the end of the $14 \mathrm{TeV}$ LHC Run, $\mathcal{L}=3000 \mathrm{fb}^{-1}$, and the estimate collected luminosity for the $100 \mathrm{TeV}$ machine of $\mathcal{L}=10 \mathrm{ab}^{-1}$. This number of events is then to be compared to the minimum number of events required to obtain $4 \sigma$ statistical significance as calculated in [9]. In Ref. [9], this significance was estimated to lead to about 20 events for $m_{A} \approx 200 \mathrm{GeV}$ and 10 events for $m_{A} \approx 400 \mathrm{GeV}$.

In Fig. 10, we show the number of raw events Eq. (14) for the $14 \mathrm{TeV}$ LHC with $3000 \mathrm{fb}^{-1}$ integrated luminosity (left panel) and for a $100 \mathrm{TeV}$ hadron machine with $10 \mathrm{ab}^{-1}$ luminosity (right panel). It is clear that even with the high luminosity option at the LHC, the golden mode could only be probed, if at all, in a very narrow region of the $2 \mathrm{HDM}$ parameter space where $m_{A} \approx 2 m_{t}$ which is due to $t \bar{t}$ threshold effect. Furthermore, this number of events is obtained for $\tan \beta=1$, and above that value it is hopeless to expect any significant number of events. Of course, if the bounds would allow to lower $\tan \beta$ to values below 1 , the number of events would grow. However, present and future constraints on the $2 \mathrm{HDM}$ are moving the parameter space further and further away from the small $\tan \beta$ values. Moving to a $100 \mathrm{TeV}$ machine it is clear that the golden mode could be probed with an integrated luminosity of

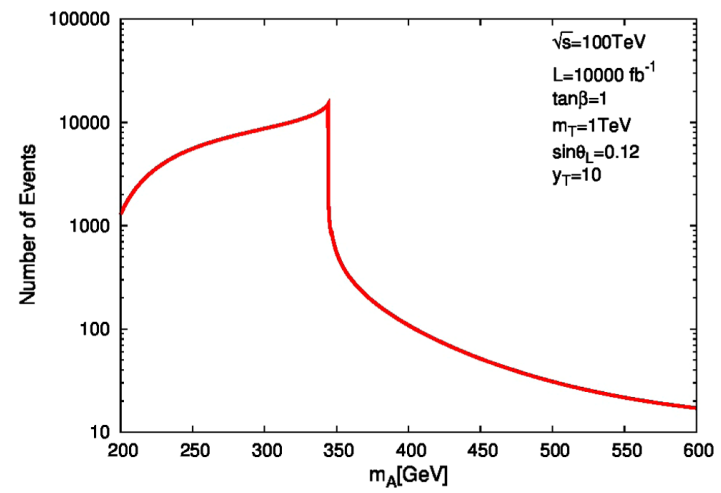

FIG. 11. Number of events obtained at the $14 \mathrm{TeV}$ LHC with a total integrated luminosity of $3000 \mathrm{fb}^{-1}$ (left) and at a $100 \mathrm{TeV}$ machine with $10 \mathrm{ab}^{-1}$ integrated luminosity for the $2 \mathrm{HDM}+\mathrm{T}$. We assume that the efficiency factor is $\epsilon \approx 0.35$. 
$10 \mathrm{ab}^{-1}$ in the $C P$-odd mass range of 200 to $400 \mathrm{GeV}$. In this mass range, the total number of events produced would be in the range of roughly 100 to 6000 events. In the $2 \mathrm{HDM}+\mathrm{T}$ (Fig. 11), the number of events is larger than for the $2 \mathrm{HDM}$ but again it is in the range of 200 to $400 \mathrm{GeV}$ that the model can be probed. Two final comments are in order. First, it is clear that at the end of the present LHC run and even more at the end of the high luminosity phase, the $2 \mathrm{HDM}+\mathrm{T}$ will be very similar to the $2 \mathrm{HDM}$, because if nothing is found, the bound on the vector top will grow. At the same time also the 2HDM will be closer to the alignment limit. Second, if a pseudoscalar is in the mass range of 200 to $400 \mathrm{GeV}$ it will certainly be first discovered in another channel.

Here we should add that although there is no estimate, the 2HDM-T will be closer and closer to the 2HDM if nothing is found because the mass of the new vector quark will have to be larger and larger.

\section{MIMICKING A CP-VIOLATING 2HDM}

In this work, we are considering a scenario where $\sin (\beta-\alpha)=1$ (the alignment limit), the lightest scalar is the SM-like Higgs with a mass $m_{h}=125 \mathrm{GeV}$ and $m_{H^{+}}=m_{H}=600 \mathrm{GeV}$. In this scenario, the pseudoscalar will decay mainly to fermions. In the right panel of Fig. 8, we present the partial width $\Gamma(A \rightarrow Z Z)$ as a function of $m_{A}$ for the type I model for values of $\tan \beta$ between 1 and 40 . We see that below the $t \bar{t}$ threshold, where $\sigma(p p \rightarrow$ $A) \operatorname{BR}(A \rightarrow Z Z)$ is largest, the width is always below $10^{-5} \mathrm{GeV}$. In the alignment limit, $\Gamma(H \rightarrow Z Z)$ is zero at tree-level. However, using the prescription in [96] it can be shown that when the tree-level coupling $g_{H Z Z}$ is zero the one loop $\Gamma(H \rightarrow Z Z)$ is of the order $10^{-5}$ to $10^{-4}$. This means that the $\operatorname{BR}(A \rightarrow Z Z)$ and the $\operatorname{BR}(H \rightarrow Z Z)$ will be of the same order of magnitude. Even if $\operatorname{BR}(H \rightarrow Z Z)$ can be slightly larger, also the production cross section of a pseudoscalar is larger than that of a scalar in gluon fusion. Hence, if it is true that a $100 \mathrm{TeV}$ collider will be able to probe very small branching ratios of scalars to $Z Z$ it will most certainly be unable to tell the $C P$ number of the new scalar particles. Furthermore, in the alignment limit the branching ratio for the decay $A \rightarrow h Z$ is exactly zero. However, when moving away from this limit $\operatorname{BR}(A \rightarrow h Z)$ will again be small but nonzero. So, if a scalar particle is detected can we probe its $C P$ ?

As discussed in the introduction, both the $C P$-conserving and the $C P$-violating 2HDM have the same Lorentz structure in the Higgs couplings to massive gauge bosons. Therefore, angular distributions in the final states of $Z Z$ or $W W$ (four leptons) or in Higgs associated production, will not distinguish $C P$-conserving from $C P$-violating extensions such as the $2 \mathrm{HDM}$ and C2HDM. If more than one scalar is found then it is possible to search for signals of $C P$ violation at the LHC with a combination of three decays. In Ref. [17], it was shown that three simultaneous decays of
Higgs such as $h_{2} \rightarrow h_{1} Z, h_{1} \rightarrow Z Z$ and $h_{2} \rightarrow Z Z$ are a sign of $C P$ violation in any model. Also $h_{1} \rightarrow Z Z, h_{2} \rightarrow Z Z$ and $h_{3} \rightarrow Z Z$, where $h_{1}, h_{2}, h_{3}$ are generic Higgs bosons, is a clear signal of $C P$ violation in the $2 \mathrm{HDM}$ except if there is a $C P$-odd state that decays to $Z Z$ with a significant rate. That is, the combination of these three last decays can distinguish a $C P$-conserving $2 \mathrm{HDM}$ from a $C P$-violating one. Also the simultaneous processes $p p \rightarrow Z h_{1}, Z h_{2}, Z h_{3}$ was shown to be a sign of $C P$ violation in $[97,98]$. Note however that there are many models with $3 C P$-even scalars that can decay to $Z Z$, like the singlet extension or the $2 \mathrm{HDM}$ extended with a singlet. However, when the rate of $h_{i} \rightarrow Z Z$ becomes too small we no longer know if this is just a very suppressed tree-level process or one that only appears at one loop, as happens for $A \rightarrow Z Z$. As discussed in the introduction, a different manifestation of the same phenomena is through the measurement of anomalous $\mathrm{ZZZ}$ couplings. At the moment there are no predictions for these measurements at $100 \mathrm{TeV}$. However, as discussed in detail in the introduction, if no new scalars are found, and because the measurement of $f_{4}$ is still orders of magnitude away from the maximal calculated values in extensions of the SM, it should be clear that processes involving Higgs and gauge bosons either at tree-level or in loops cannot be used to probe the $C P$ of the scalars.

In conclusion, if a new scalar (and only one) is found at these very low rates, great precision is needed both from the experimental side and from the theoretical side with the calculation of higher-order corrections. But more important is that it becomes imperative to study the Higgs Yukawa couplings of the models. While in the 2HDM, the Yukawa couplings are either just a constant (scalar) or proportional to $\gamma_{5}$ (pseudoscalar), in the C2HDM the Yukawa couplings have the form $g_{\mathrm{SM}}^{h \bar{f}_{i} f_{i}}\left(a_{i}+i b_{i} \gamma_{5}\right)$, where $g_{\mathrm{SM}}^{h \bar{f}_{i} f_{i}}$ is the SM coupling for fermion $i$, and $i=U, D, L,(U, D, L$ stand for up-quarks, down-quarks and lepton couplings). Let us define the angle that measures the relative strength of pseudoscalar to scalar Yukawa component, $\phi_{i}$, as

$$
\tan \phi_{i}=b_{i} / a_{i} \quad i=U, D, L,
$$

which could in principle be measured in direct experiments at the LHC. The ATLAS and CMS collaborations have not started any direct measurements of $C P$ violation despite many proposals from the theoretical community. These proposals all have in common the need for high or even very high luminosity at the LHC and focus mainly on the tth and on the $\tau^{+} \tau^{-} h$ couplings. Measurement of $b_{U} / a_{U}$ were proposed in the process $p p \rightarrow t \bar{t} h$ in [99] and several proposals followed as for instance the ones in [100,101]. These use $C P$-odd variables together with asymmetries. Other proposals to probe the $C P$-nature of a scalar in the $t$ th vertex include the process $p p \rightarrow h j j$ [102] as first proposed in [103] and again more recently in [104], where an exclusion of $\phi_{t}>40^{\circ}\left(\phi_{t}>25^{\circ}\right)$ for an integrated 
luminosity of $50 \mathrm{fb}^{-1}\left(300 \mathrm{fb}^{-1}\right)$ was obtained for $14 \mathrm{TeV}$ and assuming $\phi_{t}=0$ as the null hypothesis. There are also a number of studies for the $\tau^{+} \tau^{-} h$ vertex [105-107] and a detailed study taking into account the main backgrounds $[108,109]$ lead to an estimate in the precision of $\Delta \phi_{\tau}$ of $15^{\circ}\left(9^{\circ}\right)$ for a luminosity of $150 \mathrm{fb}^{-1}\left(500 \mathrm{fb}^{-1}\right)$ and a center of mass energy of $14 \mathrm{TeV}$. These studies show that even for a Higgs with a mass of $125 \mathrm{GeV}$ and SM-like couplings very high luminosities are needed. An educated guess ${ }^{1}$ for a $100 \mathrm{TeV}$ pp collider would be to attain $\Delta \phi_{\tau} \sim$ $1^{\circ}$ to $2^{\circ}$ with an integrated luminosity of $10 \mathrm{ab}^{-1}$. The crucial thing is to keep control of the systematic uncertainties. Still, it is expectable that for a heavier Higgs the prospects will be much worse even if only because of the lower production cross section.

\section{CONCLUSIONS}

In this work, we have analyzed the detection of a pseudoscalar produced in gluon fusion plus $b \bar{b}$ initiated process and decaying to a pair of gauge bosons. We worked in the alignment limit of the $2 \mathrm{HDM}$, where $\sin (\beta-\alpha)=1$, driven by the precision measurements of the Higgs couplings that have shown that one of the scalars has to resemble the SM Higgs. The experimental search for a generic scalar particle at the LHC Run 1 has been already performed by ATLAS and CMS. A small portion of parameter space of the 2HDM has already been probed in the search with twophotons in the final state. For the case of the final states with two massive gauge bosons we are still at least 1 order of magnitude away from highest possible rates in the model.

We have also analyzed the 2HDM model with an extra vectorlike quark, $2 \mathrm{HDM}+\mathrm{T}$. Due to the extra loop contribution from the top partner we can have an enhancement of both the production cross section and of the decay widths. In fact, we have shown that the results for $p p \rightarrow$ $A \rightarrow \gamma \gamma$ already exclude a substancial region of the parameter space below the $t \bar{t}$ threshold. As for the decays to massive vector bosons the enhancement is not enough to reach the exclusion limit obtained during Run 1.

We have shown that in a future $100 \mathrm{TeV}$ collider with a luminosity of $10-20 \mathrm{ab}^{-1}$ almost all parameter space of four 2HDM Yukawa types will be probed in the case of the decay $A \rightarrow \gamma \gamma$. However, for the pseudoscalar decays into massive gauge bosons, possibly only a small portion of the parameter space will be at experimental reach. Also it is important to note that when all rates in the $Z Z$ final state become very small it will be extremely hard to use them to search for $C P$ violation. In the case of the $2 \mathrm{HDM}+\mathrm{T}$, as the rates are much higher, it is expected that a larger region of

\footnotetext{
${ }^{1}$ Werner Bernreuther, private communication.
}

the parameter space will be probed. By the end of Run 2, and if no new physics is found, the model will be closer to alignment, and the limits on heavy scalars will be stronger. In that case, the $100 \mathrm{TeV}$ collider will start operation with severe constraints both on the Higgs couplings and on the masses/couplings of extra scalars. Note that in the limit where the new top decouples, the results are similar to the 2HDM ones.

One of the main ideas that triggered this work was the search for $C P$ violation. In the $C P$-violating $2 \mathrm{HDM}$, the decays of any scalar to $Z Z$ are allowed. However, a $C P$-odd particle cannot decay to $Z Z$ at tree-level. We have shown that for the $2 \mathrm{HDM}$ the process $A \rightarrow Z Z$ is of the same order of magnitude as $H \rightarrow Z Z$ if the tree-level $H Z Z$ coupling is zero, that is, if we are close to aligment. If such a final state is detected at Run 2 or at a future $100 \mathrm{TeV}$ collider with a very low rate, it will be very hard to conclude anything about $C P$ violation. If a new scalar is detected in this final state with a higher rate it can then be a scalar from a $2 \mathrm{HDM}$ but also a pseudoscalar from an extended version of the $2 \mathrm{HDM}$, the $2 \mathrm{HDM}+\mathrm{T}$. Hence, and fortunately, a lot of work is expected to pinpoint the underlying model. The $C P$ nature of any new scalar will for sure have to rely on direct measurements of the ratio of pseudoscalar to scalar components in the Yukawa couplings. Very preliminary studies have been performed for a scalar decaying into $\tau^{+} \tau^{-}$ and in $t$ th production.

Finally we note that many other models with an extended Higgs sector will behave exactly like the 2HDM. In fact, if we extend the 2HDM with a singlet we end up with a model that in the alignment limit has a pseudoscalar that couples to the remaining SM particles exactly like the 2HDM. Therefore, our conclusions are valid for all extensions of the SM where alignment leads to a pseudoscalar with 2HDM-like couplings.

\section{ACKNOWLEDGMENTS}

The authors are supported by Grant H2020-MSCARISE-2014 No. 645722 (NonMinimalHiggs). This work is also supported by the Moroccan Ministry of Higher Education and Scientific Research MESRSFC and CNRST: Project No. PPR/2015/6. J. E. would like to thank Shaaban Khalil for the hospitality extended to him during his stay at the Center for Fundamental Physics (CFP) at Zewail City of Science and Technology where part of this work was done. He also acknowledges the receipt of the grant from the Abdus Salam International Center for Theoretical Physics, Trieste, Italy. R. S. is also supported in part by the National Science Centre, Poland, the HARMONIA project under Contract No. UMO-2015/18/ M/ST2/00518. We acknowledge discussions with Nikos Rompotis, Werner Bernreuther, and Ritesh Singh. 
[1] G. Aad et al. (ATLAS Collaboration), Phys. Lett. B 716, 1 (2012).

[2] S. Chatrchyan et al. (CMS Collaboration), Phys. Lett. B 716, 30 (2012).

[3] D. de Florian et al. (LHC Higgs Cross Section Working Group), arXiv:1610.07922.

[4] T. D. Lee, Phys. Rev. D 8, 1226 (1973).

[5] A. Arhrib and R. Benbrik, arXiv:hep-ph/0610184.

[6] J. L. Diaz-Cruz, C. G. Honorato, J. A. Orduz-Ducuara, and M. A. Perez, Phys. Rev. D 90, 095019 (2014).

[7] D. Chowdhury and O. Eberhardt, J. High Energy Phys. 05 (2018) 161.

[8] W. Bernreuther, P. Gonzalez, and M. Wiebusch, Eur. Phys. J. C 69, 31 (2010).

[9] J. F. Gunion, H. E. Haber, and C. Kao, Phys. Rev. D 46, 2907 (1992).

[10] J. A. Aguilar-Saavedra, R. Benbrik, S. Heinemeyer, and M. Pérez-Victoria, Phys. Rev. D 88, 094010 (2013).

[11] G. Cacciapaglia, A. Deandrea, N. Gaur, D. Harada, Y. Okada, and L. Panizzi, J. High Energy Phys. 09 (2015) 012.

[12] A. Arhrib, R. Benbrik, S. J. D. King, B. Manaut, S. Moretti, and C. S. Un, Phys. Rev. D 97, 095015 (2018).

[13] G. Aad et al. (ATLAS Collaboration), Phys. Lett. B 726, 120 (2013).

[14] A. M. Sirunyan et al. (CMS Collaboration), Phys. Lett. B 775, 1 (2017).

[15] S. Y. Choi, D. J. Miller, M. M. Muhlleitner, and P. M. Zerwas, Phys. Lett. B 553, 61 (2003).

[16] C. P. Buszello, I. Fleck, P. Marquard, and J. J. van der Bij, Eur. Phys. J. C 32, 209 (2004).

[17] D. Fontes, J. C. Romão, R. Santos, and J. P. Silva, Phys. Rev. D 92, 055014 (2015).

[18] K. J. F. Gaemers and G. J. Gounaris, Z. Phys. C 1, 259 (1979).

[19] K. Hagiwara, R. D. Peccei, D. Zeppenfeld, and K. Hikasa, Nucl. Phys. B282, 253 (1987).

[20] G. J. Gounaris, J. Layssac, and F. M. Renard, Phys. Rev. D 61, 073013 (2000).

[21] G. J. Gounaris, J. Layssac, and F. M. Renard, Phys. Rev. D 65, 017302 (2001); 62, 073012 (2000).

[22] B. Grzadkowski, O. M. Ogreid, and P. Osland, J. High Energy Phys. 05 (2016) 025; 11 (2017) 002(E).

[23] H. Belusca-Maito, A. Falkowski, D. Fontes, J. C. Romão, and J. P. Silva, J. High Energy Phys. 04 (2018) 002.

[24] D. Fontes, M. Mühlleitner, J. C. Romão, R. Santos, J. P. Silva, and J. Wittbrodt, J. High Energy Phys. 02 (2018) 073.

[25] D. Azevedo, P. M. Ferreira, M. M. Muhlleitner, S. Patel, R. Santos, and J. Wittbrodt, J. High Energy Phys. 11 (2018) 091.

[26] G. Aad et al. (ATLAS Collaboration), J. High Energy Phys. 03 (2013) 128.

[27] V. Khachatryan et al. (CMS Collaboration), Phys. Lett. B 740, 250 (2015); 757, 569(E) (2016).

[28] V. Khachatryan et al. (CMS Collaboration), Eur. Phys. J. C 75, 511 (2015).

[29] R. Rahaman and R. K. Singh, arXiv:1810.11657.

[30] P. M. Ferreira, R. Santos, and A. Barroso, Phys. Lett. B 603, 219 (2004); 629, 114(E) (2005).
[31] A. Barroso, P. M. Ferreira, and R. Santos, Phys. Lett. B 632, 684 (2006).

[32] A. Barroso, P. M. Ferreira, I. P. Ivanov, and R. Santos, J. High Energy Phys. 06 (2013) 045.

[33] I. P. Ivanov, Phys. Rev. E 79, 021116 (2009).

[34] S. L. Glashow and S. Weinberg, Phys. Rev. D 15, 1958 (1977).

[35] E. A. Paschos, Phys. Rev. D 15, 1966 (1977).

[36] N. G. Deshpande and E. Ma, Phys. Rev. D 18, 2574 (1978).

[37] I. P. Ivanov, Phys. Rev. D 75, 035001 (2007); 76, 039902 (E) (2007).

[38] G. C. Branco, P. M. Ferreira, L. Lavoura, M. N. Rebelo, M. Sher, and J. P. Silva, Phys. Rep. 516, 1 (2012).

[39] S. Kanemura, T. Kubota, and E. Takasugi, Phys. Lett. B 313, 155 (1993).

[40] A. G. Akeroyd, A. Arhrib, and E.-M. Naimi, Phys. Lett. B 490, 119 (2000).

[41] CDF, DELPHI, ALEPH, SLD, OPAL, D0, and L3 Collaborations, Tevatron Electroweak Working, SLD Electroweak and Heavy Flavour, LEP Electroweak Working, and L. E. W. Groups, arXiv:1012.2367.

[42] M. E. Peskin and T. Takeuchi, Phys. Rev. D 46, 381 (1992).

[43] C. D. Froggatt, R. G. Moorhouse, and I. G. Knowles, Phys. Rev. D 45, 2471 (1992).

[44] W. Grimus, L. Lavoura, O. M. Ogreid, and P. Osland, Nucl. Phys. B801, 81 (2008).

[45] H. E. Haber and D. O’Neil, Phys. Rev. D 83, 055017 (2011).

[46] M. Baak, J. Haller, A. Hoecker, D. Kennedy, K. Mönig, M. Schott, and J. Stelzer, Eur. Phys. J. C 72, 2003 (2012).

[47] P. Bruckman De Renstrom (ATLAS and CMS Collaborations), in Proceedings, 3rd Large Hadron Collider Physics Conference (LHCP 2015): St. Petersburg, Russia, 2015, (Kurchatov Institute, Gatchina, 2016), pp. 192-206.

[48] A. Arhrib, K. Cheung, J. S. Lee, and C.-T. Lu, J. High Energy Phys. 05 (2016) 093.

[49] A. M. Sirunyan et al. (CMS Collaboration), J. High Energy Phys. 09 (2018) 007.

[50] A. Wahab El Kaffas, P. Osland, and O. M. Ogreid, Phys. Rev. D 76, 095001 (2007).

[51] M. Aoki, S. Kanemura, K. Tsumura, and K. Yagyu, Phys. Rev. D 80, 015017 (2009).

[52] S. Su and B. Thomas, Phys. Rev. D 79, 095014 (2009).

[53] F. Mahmoudi and O. Stal, Phys. Rev. D 81, 035016 (2010).

[54] O. Deschamps, S. Monteil, V. Niess, S. Descotes-Genon, S. T'Jampens, and V. Tisserand, Phys. Rev. D 82, 073012 (2010).

[55] A. Denner, R. J. Guth, W. Hollik, and J. H. Kuhn, Z. Phys. C 51, 695 (1991).

[56] H. E. Haber and H. E. Logan, Phys. Rev. D 62, 015011 (2000).

[57] A. Freitas and Y.-C. Huang, J. High Energy Phys. 08 (2012) 050; 10 (2013) 044(E).

[58] M. Misiak et al., Phys. Rev. Lett. 114, 221801 (2015).

[59] M. Misiak and M. Steinhauser, Eur. Phys. J. C 77, 201 (2017).

[60] S. Chatrchyan et al. (CMS Collaboration), J. High Energy Phys. 07 (2012) 143. 
[61] G. Aad et al. (ATLAS Collaboration), J. High Energy Phys. 06 (2012) 039.

[62] G. Abbiendi et al. (LEP, DELPHI, OPAL, ALEPH, and L3 Collaborations), Eur. Phys. J. C 73, 2463 (2013).

[63] S. P. Martin, Phys. Rev. D 81, 035004 (2010).

[64] K. Kong, S. C. Park, and T. G. Rizzo, J. High Energy Phys. 07 (2010) 059.

[65] N. Arkani-Hamed, A. G. Cohen, E. Katz, and A. E. Nelson, J. High Energy Phys. 07 (2002) 034.

[66] D. B. Kaplan, H. Georgi, and S. Dimopoulos, Phys. Lett. 136B, 187 (1984).

[67] A. Angelescu, A. Djouadi, and G. Moreau, Eur. Phys. J. C 76, 99 (2016).

[68] G. Aad et al. (ATLAS Collaboration), J. High Energy Phys. 10 (2015) 150.

[69] G. Aad et al. (ATLAS Collaboration), J. High Energy Phys. 08 (2015) 105.

[70] V. Khachatryan et al. (CMS Collaboration), Phys. Rev. D 93, 012003 (2016).

[71] M. Aaboud et al. (ATLAS Collaboration), Phys. Rev. D 94, 052009 (2016).

[72] M. Aaboud et al. (ATLAS Collaboration), J. High Energy Phys. 08 (2017) 052.

[73] A. M. Sirunyan et al. (CMS Collaboration), Phys. Lett. B 779, 82 (2018).

[74] M. Aaboud et al. (ATLAS Collaboration), J. High Energy Phys. 10 (2017) 141.

[75] G. Aad et al. (ATLAS Collaboration), Phys. Rev. D 86, 012007 (2012).

[76] A. Angelescu, A. Djouadi, and G. Moreau, Phys. Lett. B 756, 126 (2016).

[77] J. A. Aguilar-Saavedra, D. E. López-Fogliani, and C. Muñoz, J. High Energy Phys. 06 (2017) 095.

[78] R. V. Harlander, S. Liebler, and H. Mantler, Comput. Phys. Commun. 184, 1605 (2013).

[79] A. Djouadi, J. Kalinowski, and M. Spira, Comput. Phys. Commun. 108, 56 (1998).

[80] A. Djouadi, M. M. Muhlleitner, and M. Spira, Acta Phys. Pol. B 38, 635 (2007).

[81] A. Djouadi, J. Kalinowski, M. Muehlleitner, and M. Spira, arXiv:1801.09506.

[82] R. Harlander, M. Mühlleitner, J. Rathsman, M. Spira, and O. Stål, arXiv:1312.5571.

[83] T. Hahn, Comput. Phys. Commun. 140, 418 (2001).

[84] T. Hahn and M. Perez-Victoria, Comput. Phys. Commun. 118, 153 (1999).

[85] G. J. van Oldenborgh and J. A. M. Vermaseren, Z. Phys. C 46, 425 (1990).
[86] G. Aad et al. (ATLAS Collaboration), Phys. Rev. Lett. 113, 171801 (2014).

[87] V. Khachatryan et al. (CMS Collaboration), Phys. Lett. B 750, 494 (2015).

[88] CMS Collaboration, CERN Report No. CMS-PAS-HIG14-037, 2015.

[89] G. Aad et al. (ATLAS Collaboration), J. High Energy Phys. 01 (2016) 032.

[90] G. Aad et al. (ATLAS Collaboration), Eur. Phys. J. C 76, 45 (2016).

[91] V. Khachatryan et al. (CMS Collaboration), J. High Energy Phys. 10 (2015) 144.

[92] P. M. Ferreira, J. F. Gunion, H. E. Haber, and R. Santos, Phys. Rev. D 89, 115003 (2014).

[93] P. M. Ferreira, R. Guedes, M. O. P. Sampaio, and R. Santos, J. High Energy Phys. 12 (2014) 067.

[94] I. Hinchliffe, A. Kotwal, M. L. Mangano, C. Quigg, and L.-T. Wang, Int. J. Mod. Phys. A 30, 1544002 (2015).

[95] J. F. Gunion, R. Bork, H. E. Haber, and A. Seiden, Phys. Rev. D 46, 2040 (1992).

[96] M. Krause, R. Lorenz, M. Muhlleitner, R. Santos, and H. Ziesche, J. High Energy Phys. 09 (2016) 143.

[97] A. G. Akeroyd and A. Arhrib, Phys. Rev. D 64, 095018 (2001).

[98] A. Arhrib, D. K. Ghosh, and O. C. W. Kong, Phys. Lett. B 537, 217 (2002).

[99] J. F. Gunion and X.-G. He, Phys. Rev. Lett. 76, 4468 (1996).

[100] F. Boudjema, R. M. Godbole, D. Guadagnoli, and K. A. Mohan, Phys. Rev. D 92, 015019 (2015).

[101] S. P. Amor dos Santos et al., Phys. Rev. D 92, 034021 (2015).

[102] V. Del Duca, W. Kilgore, C. Oleari, C. Schmidt, and D. Zeppenfeld, Nucl. Phys. B616, 367 (2001).

[103] B. Field, Phys. Rev. D 66, 114007 (2002).

[104] M. J. Dolan, P. Harris, M. Jankowiak, and M. Spannowsky, Phys. Rev. D 90, 073008 (2014).

[105] S. Berge, W. Bernreuther, and J. Ziethe, Phys. Rev. Lett. 100, 171605 (2008).

[106] R. Harnik, A. Martin, T. Okui, R. Primulando, and F. Yu, Phys. Rev. D 88, 076009 (2013).

[107] A. Askew, P. Jaiswal, T. Okui, H. B. Prosper, and N. Sato, Phys. Rev. D 91, 075014 (2015).

[108] S. Berge, W. Bernreuther, and S. Kirchner, Eur. Phys. J. C 74, 3164 (2014).

[109] S. Berge, W. Bernreuther, and S. Kirchner, Phys. Rev. D 92, 096012 (2015). 